\title{
THE EFFECT OF THE GRAPH TOPOLOGY ON THE EXISTENCE OF MULTIPEAK SOLUTIONS FOR NONLINEAR SCHRÖDINGER EQUATIONS
}

\author{
E. N. DANCER, K. Y. LAM AND S. YAN
}

\section{INTRODUCTION}

Consider

$$
\begin{cases}-\varepsilon^{2} \Delta u+V(y) u=u^{p-1}, & y \in R^{N}, \\ u>0, & y \in R^{N}, \\ u \rightarrow 0, & \text { as }|y| \rightarrow+\infty,\end{cases}
$$

where $V(y)$ is a smooth bounded function with positive lower bound, $\varepsilon>0$ is a small number, $2<p<\frac{2 N}{N-2}$ if $N>2$ and $2<p<+\infty$ if $N=2$.

Many works have been done on problem (1.1) recently. See for example $[6,7,8,16,21,22,23]$. One of the results in the papers just mentioned is that if $x_{1}, x_{2}, \ldots, x_{k}$ are $k$ different strictly local minimum points of $V(y)$, then (1.1) has a $k$-peak solution $u_{\varepsilon}$, that is, solution with exactly $k$ local maximum points, such that $u_{\varepsilon}$ has exactly one local maximum point in a neighbourhood of $x_{j}, j=1, \ldots, k$. The same conclusion is also true if $x_{1}, x_{2}, \ldots, x_{k}$ are $k$ different strictly local maximum points of $V(y)$. Actually, it is proved in [23] that (1.1) has a multipeak solution with all its peaks near an isolated maximum point of $V(y)$. Thus a natural question is what will happen if $V(y)$ attains its local minimum or local maximum on a connected set. Especially, if $V(y)$ attains its local minimum on a connected set which contains infinitely many points, it is interesting to study whether (1.1) has a multipeak solution concentrating on this set. Generally, this is not true as shown by example (1.6).

1991 Mathematics Subject Classification. 35B25, 35J25, 58E05.

Key words and phrases. Graph topology, multipeak solutions, nonlinear Schrödinger equations.

This work was supported by the ARC.

Received: September 16, 1998. 
The main results of this paper consist of three parts. Firstly, we study how the topological structure of the local minimum set of the potential $V(y)$ affects the existence of multipeak solutions for (1.1). We will show that if the minimum set of $V(y)$ has nontrivial reduced homology, then for each $k \geq 1$, (1.1) has at least one $k$-peak solution such that each local maximum point of this solution tends to a point in this minimum set as $\varepsilon \rightarrow 0$.

Secondly, we construct solutions with their peaks near a connected maximum set of $V(y)$. Unlike the the case of minimum sets, we show that for any connected maximum set of $V(y)$ and for any positive integer $k \geq 2$, (1.1) always has at least $N$ different solutions with all their peaks tending to this maximum set as $\varepsilon \rightarrow 0$.

Suppose that $M_{1}$ and $M_{2}$ are two disjoint connected sets such that $V(y)$ attains a local minimum or a local maximum on $M_{1}$ and on $M_{2}$. The third problem studied in this paper is to construct a $\left(k_{1}+k_{2}\right)$-peak solution $u_{\varepsilon}$ such that $u_{\varepsilon}$ has exactly $k_{i}$ local maximum points near $M_{i}, i=1,2$.

Before we state our results precisely, we give some notation first.

For any constant $\bar{V}>0$, let $U_{\bar{V}}(y)$ be the unique solution of

$$
\begin{cases}-\Delta u+\bar{V} u=u^{p-1}, & y \in R^{N}, \\ u>0, & y \in R^{N}, \\ u \in H^{1}\left(R^{N}\right), & \\ u(0)=\max _{y \in R^{N}} u(y) . & \end{cases}
$$

Let $w$ be the unique solution of

$$
\begin{cases}-\Delta u+u=u^{p-1}, & y \in R^{N}, \\ u>0, & y \in R^{N}, \\ u \in H^{1}\left(R^{N}\right), & \\ u(0)=\max _{y \in R^{N}} u(y) . & \end{cases}
$$

Then $U_{\bar{V}}(y)=\bar{V}^{\frac{1}{p-2}} w(\bar{V} y)$. Denote $U_{\varepsilon, z, \bar{V}}(y)=U_{\bar{V}}\left(\frac{y-z}{\varepsilon}\right)$.

For any fixed integer $k>0$ and $V_{j}>0, j=1, \cdots, k$, we denote

$$
\begin{gathered}
E_{\varepsilon, x, k}=\left\{v \in H^{1}\left(R^{N}\right):\left\langle U_{\varepsilon, x_{j}, V_{j}}, v\right\rangle_{\varepsilon}=\left\langle\frac{\partial U_{\varepsilon, x_{j}, V_{j}}}{\partial x_{j i}}, v\right\rangle_{\varepsilon}=0,\right. \\
j=1, \ldots, k, i=1, \ldots, N\},
\end{gathered}
$$

where $\langle u, v\rangle_{\varepsilon}=\int_{R^{N}} \varepsilon^{2} D u D v+V(y) u v$. We also denote $\|v\|_{\varepsilon}^{2}=\int_{R^{N}} \varepsilon^{2}|D u|^{2}+$ $V(y) v^{2}$.

Definition 1.1. Let $M$ be a connected compact set in $R^{N}$. $M$ is said to be a local minimum (maximum) set of $V(y)$ if there are constants $\gamma>0$ and $V_{M}$, such that $V(y)=V_{M}$ for $y \in M, V(y)>V_{M}\left(V(y)<V_{M}\right)$ for $y \in M_{\gamma} \backslash M$, where $M_{\gamma}=\left\{z: z \in R^{N}, d(z, M) \leq \gamma\right\}$.

Throughout this paper, all the homologies are with $Z_{2}$-coefficients. Now we are ready to state our main results. 
Theorem 1.2. Suppose that $M$ is a connected compact local minimum set of $V(y)$ such that $\partial M_{\gamma}$ is a $C^{1}$ manifold of dimension $N-1$ for each $\gamma>$ 0 small. Moreover, we assume that for any $\gamma>0$ small, the following conditions hold:

$$
V(y) \leq V_{M}+a d(y, M)^{h}, \quad\left|D^{m} V(y)\right|=O\left(d(y, M)^{h-m}\right),
$$

for $m=1, \ldots,[h], y \in M_{\gamma}$,

$$
\langle D V(y), n\rangle \geq c_{0} \gamma^{h-1}, \forall y \in \partial M_{\gamma},
$$

where $a, c_{0}$ and $h \geq 2$ are some positive constants, $n$ is the outward unit normal of $\partial M_{\gamma}$ at $y$. If the reduced homology of $M$ is nontrivial, then for each integer $k \geq 2$, there is an $\varepsilon_{0}>0$, such that for every $\varepsilon \in\left(0, \varepsilon_{0}\right],(1.1)$ has at least one $k$-peak solution of the form

$$
u_{\varepsilon}=\sum_{j=1}^{k} \alpha_{\varepsilon, j} U_{\varepsilon, x_{\varepsilon, j}, V_{M}}+v_{\varepsilon},
$$

where $v_{\varepsilon} \in E_{\varepsilon, x, k}$ and as $\varepsilon \rightarrow 0$,

$$
\alpha_{\varepsilon, j} \rightarrow 1, \frac{\left|x_{\varepsilon, i}-x_{\varepsilon, j}\right|}{\varepsilon} \rightarrow \infty, x_{\varepsilon, j} \rightarrow x_{j} \in M,\left\|v_{\varepsilon}\right\|_{\varepsilon}^{2}=o\left(\varepsilon^{N}\right),
$$

for $i, j=1, \cdots, k$ and $i \neq j$. Moreover, if $k=2$, (1.1) has at least cuplength $(M)$ distinct solutions of the form (1.6) satisfying (1.7).

For any set $M$, denote

$$
A_{k}=(\underbrace{M_{\gamma} \times \cdots \times M_{\gamma}}_{k} \backslash \bigcup_{i \neq j}\left\{\left|x_{i}-x_{j}\right|<d\right\}) / \sigma_{k},
$$

where $d>0$ and $\gamma>0$ are small constants, $\sigma_{k}$ is the group of permutations of $k$ letters acting on $\underbrace{M_{\gamma} \times \cdots \times M_{\gamma}}_{k} \backslash \bigcup_{i \neq j}\left\{\left|x_{i}-x_{j}\right|<d\right\}$.

Theorem 1.3. Suppose that $M$ is a connected compact local maximum set of $V(x)$. Then for each positive integer $k \geq 2$, there is an $\varepsilon_{0}>0$, such that for every $\varepsilon \in\left(0, \varepsilon_{0}\right],(1.1)$ has at least Cat $_{A_{k}}\left(A_{k}\right)$ solutions of the form

$$
u_{\varepsilon}=\sum_{j=1}^{k} \alpha_{\varepsilon, j} U_{\varepsilon, x_{\varepsilon, j}, V_{M}}+v_{\varepsilon},
$$

where $v_{\varepsilon} \in E_{\varepsilon, x, k}$ and as $\varepsilon \rightarrow 0$,

$$
\alpha_{\varepsilon, j} \rightarrow 1, \frac{\left|x_{\varepsilon, i}-x_{\varepsilon, j}\right|}{\varepsilon} \rightarrow \infty, x_{\varepsilon, j} \rightarrow x_{j} \in M,\left\|v_{\varepsilon}\right\|_{\varepsilon}^{2}=o\left(\varepsilon^{N}\right) .
$$

for $i, j=1, \cdots, k$ and $i \neq j$.

Theorem 1.4. Let $M_{1}, \ldots, M_{l}$ be disjoint connected compact sets such that $\partial M_{i, \gamma}$ is a $C^{1}$-manifold of dimension $N-1$ for each $\gamma>0$ small and $i=$ $1, \cdots, l$. Suppose that the following conditions hold: there are constants $h \geq 2, c_{1} \geq c_{0}>0$ such that 
(1.10) $V_{M_{i}} \leq V(y) \leq V_{M_{i}}+c_{1} d\left(y, M_{i}\right)^{h},\left|D^{m} V(y)\right|=O\left(d\left(y, M_{i}\right)^{h-m}\right)$,

$$
\begin{gathered}
\langle D V(y), n\rangle \geq c_{0} \gamma^{h-1}, \forall y \in \partial M_{i, \gamma}, i=1, \ldots, l_{1}, \\
c_{0} d\left(y, M_{i}\right)^{h} \leq V_{M_{i}}-V(y) \leq c_{1} d\left(y, M_{i}\right)^{h}, \\
\left|D^{m} V(y)\right|=O\left(d\left(y, M_{i}\right)^{h-m}\right), \\
\forall y \in M_{i, \gamma}, m=1, \ldots,[h], i=l_{1}+1, \ldots, l .
\end{gathered}
$$

(i) If each $M_{i}, i=1, \ldots, l_{1}$, has nontrivial reduced homology, then for any positive integers $k_{i}, i=1, \ldots, l$, there is an $\varepsilon_{0}>0$ such that for each $\varepsilon \in\left(0, \varepsilon_{0}\right],(1.1)$ has at least one solution of the form

$$
u_{\varepsilon}=\sum_{i=1}^{l} \sum_{j=1}^{k_{i}} \alpha_{\varepsilon, i, j} U_{\varepsilon, x_{\varepsilon, i, j}, V_{M_{i}}}+v_{\varepsilon}
$$

where $v_{\varepsilon} \in E_{\varepsilon, x, \sum_{i=1}^{l} k_{i}}$ and as $\varepsilon \rightarrow 0$,

$$
\begin{gathered}
\alpha_{\varepsilon, i, j} \rightarrow 1, \quad\left\|v_{\varepsilon}\right\|_{\varepsilon}^{2}=o\left(\varepsilon^{N}\right), \\
\frac{\left|x_{\varepsilon, i, j}-x_{\varepsilon, i, m}\right|}{\varepsilon} \rightarrow \infty, \quad x_{\varepsilon, i, j} \rightarrow x_{i, j} \in M_{i}
\end{gathered}
$$

for $i=1, \cdots, l, j, m=1, \cdots, k_{i}$ and $j \neq m$.

(ii) If some of the minimum sets $M_{i_{1}}, \ldots, M_{i_{t}}$ have trivial reduced homology, then the conclusion in (i) holds for $k_{i_{j}}=1, j=1, \ldots, t$.

The basic idea to prove Theorem 1.2 can also be used to obtain the following result.

Theorem 1.5. Let $M$ be a connected compact local minimum or maximum set. Then there is an $\varepsilon_{0}>0$, such that for each $\varepsilon \in\left(0, \varepsilon_{0}\right],(1.1)$ has at least $\mathrm{Cat}_{M}(M)$ single peak positive solutions of the form:

$$
u_{\varepsilon}=\alpha_{\varepsilon} U_{\varepsilon, x_{\varepsilon}, V_{M}}+v_{\varepsilon},
$$

where, $v_{\varepsilon} \in E_{\varepsilon, x_{\varepsilon}, 1}$, and as $\varepsilon \rightarrow 0, \alpha_{\varepsilon} \rightarrow 1, x_{\varepsilon} \rightarrow x_{0} \in M$ and $\left\|v_{\varepsilon}\right\|_{\varepsilon}^{2}=o\left(\varepsilon^{N}\right)$.

The assumption that $M$ has nontrivial topology is essential in Theorem 1.2 , as shown by the following example.

Example 1.6. Let $V(y)=V(|y|)$ be a smooth function satisfying $V(y)=1$ for $|y| \leq 1$, and $V(|y|)$ is strictly increasing in $|y|>1$. Then we see that the minimum set of $V(y)$ is the unit ball in $R^{N}$. By the moving plane method of Gidas, Ni and Nirenberg [19], we know that every solution of (1.1) is radially symmetric and strictly decreasing. Therefore the solution has a unique maximum point, and thus is a single peak solution. 
Example 1.\%. A typical example of $V(y)$ satisfying the conditions in Theorem 1.4 is that $V(y)=V(|y|)$ and $V(r)$ attains its local minimum or maximum at $r_{l}>\cdots>r_{1}>0$ with $V^{\prime \prime}\left(r_{i}\right) \neq 0, i=1, \cdots, l$. In this case, $M_{i}=\left\{y:|y|=r_{i}\right\}, h=2$.

Remark 1.8. If $M$ is an $N$-dimensional smooth manifold without boundary, tubular neighbourhoods of $M$ are useful for verifying the conditions in Theorem 1.4.

Remark 1.9. By Proposition C.3, we know that the lower bound for $\operatorname{Cat}_{A_{k}} A_{k}$ is $N$. So (1.1) has at least $N$ different $k$-peak solutions concentrating on the connected compact local maximum set of $V(y)$.

Since the work by Bahri and Coron [2], the effect of the domain topology on the existence and multiplicity of the solutions is one of the subjects which attract much attention. See for example $[2,3,4,10,11,12,14,15]$. In [3], the category of the domain was used to estimate the number of the single peak solutions, while in $[12,14,15]$, the effect of the domain topology on the existence of multipeak solutions was studied. The domain in problem (1.1) has trivial topology, so our results here emphasize the effect of the topology of the level set of the potential $V(y)$ on the existence and multiplicity of multipeak solutions for (1.1).

Finally, let us point out that the idea in this paper works for the singularly perturbed Neumann problem:

$$
\begin{cases}-\varepsilon^{2} \Delta u+u=u^{p-1}, & \text { in } \Omega, \\ u>0, & \text { in } \Omega, \\ \frac{\partial u}{\partial n}=0, & \text { in } \partial \Omega,\end{cases}
$$

where $\Omega$ is a bounded domain in $R^{N}$. The role of the mean curvature function of the boundary $\partial \Omega$ in (1.19) is similar to that of the potential $V(x)$ in (1.1). The estimates in Appendix $\mathrm{C}$ can be used to improve the multiplicity results in $[13,25]$. For example, as a direct corollary of Proposition C.3 and the results in $[13,25]$, we have

Corollary 1.10. For each integer $k \geq 2$, there is $\varepsilon_{0}>0$ such that for each $\varepsilon \in\left(0, \varepsilon_{0}\right]$,

(i) (1.19) has at least $N$ boundary $k$-peak solutions with all their local maximum points near the global minimum set of the mean curvature function of $\partial \Omega$;

(ii) (1.19) has at least $N$ interior $k$-peak solutions.

This paper is arranged as follows. In section 2, we reduce the problem of finding a multipeak solution for (1.1) to a finite dimensional problem. Theorem 1.2 is proved in section 3 . Section 4 is devoted to the proof of Theorems 1.3 and 1.5. In section 5, we prove Theorem 1.4. Some basic estimates and topological results needed in the proof of the main results are presented in the appendices. 


\section{Reduction to Finite dimensional problem}

First we define

$$
\begin{gathered}
D_{\varepsilon}=\left\{(\alpha, x, v):\left|\alpha_{j}-1\right| \leq \delta, v \in E_{\varepsilon, x, k},\|v\|_{\varepsilon} \leq \delta \varepsilon^{N / 2},\right. \\
x=\left(x_{1}, \ldots, x_{k}\right), x_{j} \in R^{N}, \frac{\left|x_{i}-x_{j}\right|}{\varepsilon} \geq R, \\
i, j=1, \ldots, k, i \neq j\}
\end{gathered}
$$

where $\delta>0$ is a fixed small constant and $R>0$ is a fixed large constant.

We also define

$$
J(\alpha, x, v)=I\left(\sum_{j=1}^{k} \alpha_{j} U_{\varepsilon, x_{j}, V_{j}}+v\right), \quad \forall(\alpha, x, v) \in D_{\varepsilon},
$$

where

$$
I(u)=\frac{1}{2} \int_{R^{N}}\left(\varepsilon^{2}|D u|^{2}+V(y) u^{2}\right)-\frac{1}{p} \int_{R^{N}}|u|^{p} .
$$

It is well known now (see $[1,24]$ ) that if $\delta>0$ is small enough and $R>0$ is large enough,

$$
u=\sum_{j=1}^{k} \alpha_{j} U_{\varepsilon, x_{j}, V_{j}}+v,
$$

is a positive critical point of $I(u)$ if and only if $(\alpha, x, v)$ is a critical point of $J(\alpha, x, v)$ in $D_{\varepsilon}$. So we need to solve the following system:

$$
\begin{aligned}
\frac{\partial J}{\partial \alpha_{j}} & =0, \quad j=1, \ldots, k \\
\frac{\partial J}{\partial x_{j l}} & =\sum_{h=1}^{N} G_{h j}\left\langle\frac{\partial^{2} U_{\varepsilon, x_{j}, V_{j}}}{\partial x_{j h} \partial x_{j l}}, v\right\rangle_{\varepsilon}, \quad j=1, \ldots, k, l=1, \ldots, N \\
\frac{\partial J}{\partial v} & =\sum_{j=1}^{k} B_{j} U_{\varepsilon, x_{j}, V_{j}}+\sum_{j=1}^{k} \sum_{l=1}^{N} G_{j l} \frac{\partial U_{\varepsilon, x_{j}, V_{j}}}{\partial x_{j l}},
\end{aligned}
$$

for some constants $B_{j}, G_{j l} \in R, j=1, \ldots, k, l=1, \ldots, N$.

In this section, we reduce the problem of solving the system (2.3)-(2.5) to a finite dimensional problem. We need the following proposition.

Proposition 2.1. There is an $\varepsilon_{0}>0$, such that for each $\varepsilon \in\left(0, \varepsilon_{0}\right]$, there exists a unique $C^{1}-$ map $\left(\alpha_{\varepsilon}(x), v_{\varepsilon}(x)\right): R^{N k} \backslash \cup_{i \neq j}\left\{\left|x_{i}-x_{j}\right| \leq \varepsilon R\right\} \rightarrow$ $R_{+}^{k} \times H^{1}\left(R^{N}\right)$ such that $v_{\varepsilon} \in E_{\varepsilon, x, k}$, (2.3) and (2.5) hold for some constants $B_{j}$ and $G_{j l}$. Moreover, we have

$$
v_{\varepsilon}\left(\sigma_{k} x\right)=v_{\varepsilon}(x), \quad \sigma_{k} \alpha_{\varepsilon}\left(\sigma_{k} x\right)=\alpha_{\varepsilon}(x),
$$




$$
\begin{aligned}
& |\alpha-1| \varepsilon^{N / 2}+\|v\|_{\varepsilon} \\
= & O\left(\sum_{j=1}^{k}\left(\varepsilon^{\frac{N}{2}}\left|V\left(x_{j}\right)-V_{j}\right|+\sum_{m=1}^{[h]} \varepsilon^{\frac{N}{2}+m}\left|D^{m} V\left(x_{j}\right)\right|\right)\right) \\
& +O\left(\varepsilon^{N / 2} \sum_{i \neq j} w^{\frac{1+\tau}{2}}\left(\frac{\left|x_{i}-x_{j}\right|}{\varepsilon}\right)\right)
\end{aligned}
$$

and

$$
\begin{aligned}
& \varepsilon B_{j}, G_{j l} \\
= & O\left(\varepsilon \sum_{j=1}^{k}\left(\left|V\left(x_{j}\right)-V_{j}\right|+\sum_{m=1}^{[h]} \varepsilon^{m}\left|D^{m} V\left(x_{j}\right)\right|\right)\right) \\
& +O\left(\varepsilon \sum_{i \neq j} w^{\frac{1+\tau}{2}}\left(\frac{\left|x_{i}-x_{j}\right|}{\varepsilon}\right)\right),
\end{aligned}
$$

where $\tau>0$ is a fixed small constant and $[h]$ is the integer part of $h$.

Proof. We can follow the same procedure as in [5] to prove the existence part. (2.6) is a direct consequence of the fact $J(\alpha, x, v)=J\left(\sigma_{k} \alpha, \sigma_{k} x, v\right)$ and the uniqueness of $\left(\alpha_{\varepsilon}(x), v_{\varepsilon}(x)\right)$ satisfying (2.3) and (2.5). To get the estimate (2.7), we just need to use Lemma A.3. We can solve a system as in [24], pp 22-23 and use Lemma A.4 to get the estimate (2.8). Since the procedure is quite standard, we omit the details.

Let $\left(\alpha_{\varepsilon}(x), v_{\varepsilon}(x)\right)$ be the map obtained in Proposition 2.2. Define

$$
K(x)=J\left(\alpha_{\varepsilon}(x), x, v_{\varepsilon}(x)\right) .
$$

In order to solve (2.3)-(2.5), we only need to find a critical point for $K(x)$ in a suitable domain. So we need the following propositions.

Proposition 2.2. Suppose that $F(x)$ is a $C^{2}$ function defined in a bounded domain $\Omega$ of $R^{k N}$. If $F$ satisfies either $F(x)>c$ or $\frac{\partial F(x)}{\partial n}>0$ at each $x \in \partial \Omega$, where $n$ is the outward unit normal of $\partial \Omega$ at $x$, then

$$
\#\left\{x: D F(x)=0, x \in F^{c}\right\} \geq \operatorname{Cat}_{F^{c}}\left(F^{c}\right),
$$

where $F^{c}=\{x: x \in \Omega, F(x) \leq c\}$. In particular, $F(x)$ has at least one critical point in $F^{c}$.

Proof. Notice that our assumption implies that the following flow:

$$
\left\{\begin{aligned}
\frac{d x(t)}{d t} & =-D F(x(t)), \\
x(0) & =x_{0} \in F^{c}
\end{aligned}\right.
$$

does not leave $\Omega$. In fact, suppose that $x(t)$ touches the boundary at some time $t_{0}$. Since $F$ is decreasing along $x(t)$, we see $F\left(x\left(t_{0}\right)\right) \leq c$. Thus, by assumption, $\frac{\partial F\left(x\left(t_{0}\right)\right)}{\partial n}>0$, which implies that $-D F\left(x\left(t_{0}\right)\right)$ points into $\Omega$. So $x(t)$ moves into $\Omega$. Then Proposition 2.2 follows directly from the Ljusternik-Schnirelman theory. 
Proposition 2.3. Suppose that $F(x)$ is a $C^{2}$ function defined in a bounded domain $\Omega$ of $R^{k N}$. Let $c_{2}>c_{1}$ be two constants such that neither $c_{2}$ nor $c_{1}$ is a critical value of $F(x)$. If $F$ satisfies either $F(x)<c_{1}$ or $\frac{\partial F(x)}{\partial n}>0$ for each $x \in \partial \Omega$, then

$$
\#\left\{x: D F(x)=0, x \in F^{c_{2}} \backslash F^{c_{1}}\right\} \geq \operatorname{Cat}_{F^{c_{2}}}\left(F^{c_{2}}, F^{c_{1}}\right) .
$$

In particular, if $F^{c_{2}}$ cannot be deformed into $F^{c_{1}}, F$ has at least one critical point in $F^{c_{2}} \backslash F^{c_{1}}$.

Proof. Similar to Proposition 2.2, our assumption implies that the following flow:

$$
\left\{\begin{aligned}
\frac{d x(t)}{d t} & =-D F(x(t)), \\
x(0) & =x_{0} \in F^{c_{2}}
\end{aligned}\right.
$$

does not leave $\Omega$ before it reaches $F^{c_{1}}$. So Proposition 2.2 follows directly from the Ljusternik-Schnirelman theory.

\section{Multipeak solutions COnCEntrating on the minimum SET}

Suppose that $M$ is a connected compact local minimum set of $V(y)$ and $M_{\gamma}=\{y: d(y, M) \leq \gamma\}$. For $k \geq 2$, let

$$
\Omega_{\gamma}=\left\{x=\left(x_{1}, \cdots, x_{k}\right): x_{j} \in M_{\gamma}, j=1, \ldots, k, \frac{\left|x_{i}-x_{j}\right|}{\varepsilon} \geq R, \forall i \neq j\right\} .
$$

Define

$$
c_{\varepsilon, 1}=\varepsilon^{N}\left(k V_{M}^{\frac{p}{p-2}-\frac{N}{2}} A-T \varepsilon^{\alpha h}\right), c_{\varepsilon, 2}=\varepsilon^{N}\left(k V_{M}^{\frac{p}{p-2}-\frac{N}{2}} A+\eta\right),
$$

where $T>0$ is a large constant and $\alpha \in(0,1)$ is a fixed constant close to 1 . We also let

$$
K^{c}=\left\{x: x \in \Omega_{\varepsilon^{\alpha}}, K(x) \leq c\right\} .
$$

In this section, we shall apply Proposition 2.3 to prove that for $\varepsilon>0$ small, $K(x)$ has a critical point in $K^{c_{\varepsilon, 2}} \backslash K^{c_{\varepsilon, 1}}$. First, we prove

Lemma 3.1. For each $x \in \partial \Omega_{\varepsilon^{\alpha}}$, we have either $K(x)<c_{\varepsilon, 1}$, or $\frac{\partial K(x)}{\partial n}>0$. Proof. We divide the proof of this lemma into two steps.

Step 1. Suppose that $\frac{\left|x_{i}-x_{j}\right|}{\varepsilon}=R$ for some $i \neq j$. We claim $x \in K^{c_{\varepsilon, 1}}$. In fact, by using Proposition 2.1 and Lemma A.2, we obtain

$$
\begin{aligned}
& K(x)=I\left(\sum_{l=1}^{k} U_{\varepsilon, x_{l}, V_{M}}\right)+O\left(\varepsilon^{N}\left|\alpha_{\varepsilon}-1\right|^{2}+\|v\|_{\varepsilon}^{2}\right) \\
= & \varepsilon^{N} \sum_{j=1}^{k} V_{M}^{\frac{p}{p-2}-\frac{N}{2}} A-\sum_{i=1}^{k-1} \int_{R^{N}}\left(\sum_{j=i+1}^{k} U_{\varepsilon, x_{j}, V_{M}}\right)^{p-1} U_{\varepsilon, x_{i}, V_{M}} \\
& +O\left(\sum_{l=1}^{k}\left(\varepsilon^{N}\left|V\left(x_{l}\right)-V_{M}\right|+\sum_{m=1}^{[h]} \varepsilon^{N+m}\left|D^{m} V\left(x_{l}\right)\right|\right)\right. \\
& +O\left(\varepsilon^{N} \sum_{i \neq j} w^{1+\tau}\left(\frac{\left|x_{i}-x_{j}\right|}{\varepsilon}\right)\right)
\end{aligned}
$$




$$
\begin{aligned}
= & \varepsilon^{N} \sum_{j=1}^{k} V_{M}^{\frac{p}{p-2}-\frac{N}{2}} A-\sum_{i=1}^{k-1} \int_{R^{N}}\left(\sum_{j=i+1}^{k} U_{\varepsilon, x_{j}, V_{M}}\right)^{p-1} U_{\varepsilon, x_{i}, V_{M}} \\
& +O\left(\varepsilon^{N+\alpha h}+\varepsilon^{N} \sum_{i \neq j} w^{1+\tau}\left(\frac{\left|x_{i}-x_{j}\right|}{\varepsilon}\right)\right) .
\end{aligned}
$$

From (3.1), we have

$$
K(x) \leq \varepsilon^{N}\left(k V_{M}^{\frac{p}{p-2}-\frac{N}{2}} A-c w(R)\right)+O\left(\varepsilon^{N+\alpha h}\right)<c_{\varepsilon, 1} .
$$

Thus $x \in K^{c_{\varepsilon, 1}}$.

Step 2. Suppose that $x_{i} \in \partial M_{\varepsilon^{\alpha}}$ for some $i$. Without loss of generality, we assume $i=1$. We claim that either $K(x)<c_{\varepsilon, 1}$, or $\frac{\partial K}{\partial n}>0$, where $n$ is the outward unit normal of $\partial M_{\varepsilon^{\alpha}}$ at $x_{1}$.

For any $x_{j} \in M_{\varepsilon^{\alpha}}$ and $m \geq 2$, we have

$$
\left|V\left(x_{j}\right)-V_{M}\right|=O\left(\varepsilon^{\alpha h}\right)
$$

and

$$
\left|D^{m} V\left(x_{j}\right)\right| \varepsilon^{m}=O\left(d\left(x_{j}, M\right)^{h-m} \varepsilon^{m}\right)=O\left(\varepsilon^{\alpha(h-m)} \varepsilon^{m}\right)=O\left(\varepsilon^{\alpha h+2(1-\alpha)}\right) .
$$

So, by Proposition 2.1 and Lemma A.4, we have

$$
\begin{aligned}
& \frac{\partial K}{\partial x_{1 l}}=\frac{\partial J}{\partial x_{1 l}}+\left\langle\frac{\partial J}{\partial v}, \frac{\partial v}{\partial x_{1 l}}\right\rangle_{\varepsilon} \\
= & \frac{\partial J}{\partial x_{1 l}}+\sum_{j=1}^{k} \sum_{h=1}^{N} G_{h j}\left\langle\frac{\partial U_{\varepsilon, x_{j}, V_{M}}}{\partial x_{j h}}, \frac{\partial v}{\partial x_{1 l}}\right\rangle_{\varepsilon} \\
= & \frac{\partial J}{\partial x_{1 l}}-\sum_{h=1}^{N} G_{1 h}\left\langle\frac{\partial^{2} U_{\varepsilon, x_{1}, V_{M}}}{\partial x_{h 1} \partial x_{1 l}}, v\right\rangle_{\varepsilon} \\
= & \frac{\partial J}{\partial x_{1 l}}+O\left(\varepsilon^{N-1} \sum_{i \neq j} e^{-(1+\tau) \frac{\left|x_{i}-x_{j}\right|}{\varepsilon}}+\varepsilon^{N+\alpha h}\right) \\
= & c_{1} \varepsilon^{N} D_{l} V\left(x_{1}\right)-(p-1) \sum_{j=2}^{k} \int_{R^{N}} U_{\varepsilon, x_{1}, V_{M}}^{p-2} U_{\varepsilon, x_{j}, V_{M}} \frac{\partial U_{\varepsilon, x_{1}, V_{M}}}{\partial x_{1 l}} \\
& +O\left(\varepsilon^{N-1} \sum_{i \neq j} e^{-(1+\tau) \frac{\left|x_{i}-x_{j}\right|}{\varepsilon}}+\varepsilon^{N-1+\alpha h+2(1-\alpha)}\right) .
\end{aligned}
$$

Denote $\eta=\min _{j \neq i}\left|x_{i}-x_{j}\right|$. We distinguish two cases.

(i) Suppose that $U\left(\frac{\eta}{\varepsilon}\right)>L \varepsilon^{\alpha h}$, where $L>T$ is a large constant. In this case, we claim that $K(x)<c_{\varepsilon, 1}$. In fact, it follows from (3.1) that

$$
\begin{aligned}
K(x) & \leq \varepsilon^{N} k V_{M}^{\frac{p}{p-2}-\frac{N}{2}} A-c^{\prime} \varepsilon^{N} w\left(\frac{\eta}{\varepsilon}\right)+O\left(\varepsilon^{N+\alpha h}\right) \\
& \leq \varepsilon^{N} k V_{M}^{\frac{p}{p-2}-\frac{N}{2}} A-c^{\prime} L \varepsilon^{N+\alpha h}+C \varepsilon^{N+\alpha h}<c_{\varepsilon, 1},
\end{aligned}
$$

if $L>T$ is large enough. 
(ii) Suppose that $U\left(\frac{\eta}{\varepsilon}\right) \leq L \varepsilon^{\alpha h}$. In this case, we prove that $\frac{\partial K(x)}{\partial n}>0$, where $n$ is the outward unit normal of $\partial M_{\varepsilon^{\alpha}}$ at $x_{1}$. Since for any $j \neq 1$,

$$
\int_{R^{N}} U_{\varepsilon, x_{1}, V_{M}}^{p-2} U_{\varepsilon, x_{j}, V_{M}} \frac{\partial U_{\varepsilon, x_{1}, V_{M}}}{\partial x_{1 l}}=(c+o(1)) \varepsilon^{N-1} w\left(\frac{\left|x_{j}-x_{1}\right|}{\varepsilon}\right) \frac{x_{j l}-x_{1 l}}{\left|x_{j}-x_{1}\right|},
$$

and for small $\beta>1-\alpha$,

$$
\left\langle\frac{x_{j}-x_{1}}{\left|x_{j}-x_{1}\right|}, n\right\rangle \leq \varepsilon^{\beta}, \quad \forall x_{j} \in M_{\varepsilon^{\alpha}} \cap B_{\varepsilon^{\alpha}}\left(x_{1}\right),
$$

we see that

$$
\begin{aligned}
& \int_{R^{N}} U_{\varepsilon, x_{1}, V_{M}}^{p-2} U_{\varepsilon, x_{j}, V_{M}} \frac{\partial U_{\varepsilon, x_{1}, V_{M}}}{\partial n} \\
\leq & \varepsilon^{\beta}(c+o(1)) \varepsilon^{N-1} w\left(\frac{\left|x_{1}-x_{j}\right|}{\varepsilon}\right) \\
\leq & \varepsilon^{\beta}(c+o(1)) \varepsilon^{N-1} L \varepsilon^{\alpha h}, \quad \forall x_{j} \in M_{\varepsilon^{\alpha}} \cap B_{\varepsilon^{\alpha}}\left(x_{1}\right) .
\end{aligned}
$$

On the other hand, if $\left|x_{j}-x_{1}\right|>\varepsilon^{\alpha}$, then

$$
\int_{R^{N}} U_{\varepsilon, x_{1}, V_{M}}^{p-2} U_{\varepsilon, x_{j}, V_{M}} \frac{\partial U_{\varepsilon, x_{1}, V_{M}}}{\partial n}=O\left(\varepsilon^{N-1} e^{-\frac{1}{\varepsilon^{1-\alpha}}}\right)
$$

Combining (3.3), (3.5)-(3.6), we obtain

$$
\begin{aligned}
\frac{\partial K(x)}{\partial n} \geq & c_{1} \varepsilon^{N}\left\langle D V\left(x_{1}\right), n\right\rangle-\varepsilon^{\beta} L(c+o(1)) \varepsilon^{N-1+\alpha h} \\
& +O\left(\varepsilon^{N-1+\alpha h+2(1-\alpha)}\right) \\
\geq & c^{\prime} \varepsilon^{N+\alpha(h-1)}-L(c+o(1)) \varepsilon^{N-1+\alpha h+\beta}>0 .
\end{aligned}
$$

Combining Steps 1 and 2, we complete the proof of this lemma.

We are now ready to prove Theorems 1.2.

Proof. [Proof of Theorems 1.2]

In view of Lemma 3.1 and Proposition 2.3, we see

$$
\#\left\{x: D K(x)=0, x \in K^{c_{\varepsilon, 2}} \backslash K^{c_{\varepsilon, 1}}\right\} \geq \operatorname{Cat}_{\Omega_{\varepsilon^{\alpha}}}\left(K^{c_{\varepsilon, 2}}, K^{c_{\varepsilon, 1}}\right) .
$$

It is easy to check that

$$
K^{c_{\varepsilon, 2}}=\Omega_{\varepsilon^{\alpha}}
$$

On the other hand, we claim

$$
T_{C^{\prime} \varepsilon \ln \varepsilon^{-1}} \backslash T_{\varepsilon R} \subset K^{c_{\varepsilon, 1}} \subset T_{C \varepsilon \ln \varepsilon^{-1}} \backslash T_{\varepsilon R},
$$

where $T_{\tau}=\cup_{i \neq j}\left\{\left|x_{i}-x_{j}\right| \leq \tau, x_{i}, x_{j} \in M_{\varepsilon^{\alpha}}\right\}, C>c^{\prime}>0$ are some suitable constants.

In fact, it follows from Lemma A.2 that

$$
\begin{aligned}
c_{\varepsilon, 1} & =\varepsilon^{N} k V_{M}^{\frac{p}{p-2}-\frac{N}{2}} A-T \varepsilon^{N+\alpha h} \\
& >K(x)=\varepsilon^{N} k V_{M}^{\frac{p}{p-2}-\frac{N}{2}} A-\sum_{i \neq j} \int_{R^{N}} U_{\varepsilon, x_{i}, V_{M}}^{p-1} U_{\varepsilon, x_{j}, V_{M}}+O\left(\varepsilon^{N+\alpha h}\right),
\end{aligned}
$$


which implies $\left|x_{i}-x_{j}\right| \leq C \varepsilon \ln \frac{1}{\varepsilon}$ for some $i \neq j$ if $T>0$ is large enough. Thus,

$$
K^{c_{\varepsilon, 1}} \subset T_{C \varepsilon \ln \varepsilon^{-1}} \backslash T_{\varepsilon R} .
$$

On the other hand, it is easy to check

$$
T_{c^{\prime} \varepsilon \ln \varepsilon^{-1}} \backslash T_{\varepsilon R} \subset K^{c_{\varepsilon, 1}},
$$

if $c^{\prime}>0$ is small enough. So the claim follows.

Since the $T_{C \varepsilon \ln \varepsilon^{-1}} \backslash T_{\varepsilon R}$ can be deformed into $T_{C^{\prime} \varepsilon \ln \varepsilon^{-1}} \backslash T_{\varepsilon R}$, we have

$$
\operatorname{Cat}_{\Omega_{\varepsilon} \alpha}\left(K^{c_{\varepsilon, 2}}, K^{c_{\varepsilon, 1}}\right)=\operatorname{Cat}_{\Omega_{\varepsilon} \alpha}\left(\Omega_{\varepsilon^{\alpha}}, T_{c^{\prime} \varepsilon \ln \varepsilon^{-1}} \backslash T_{\varepsilon R}\right) .
$$

As a result, we have

$$
\begin{aligned}
& \quad \#\left\{x: D K(x)=0, x \in K^{c_{\varepsilon, 2}} \backslash K^{c_{\varepsilon, 1}}\right\} \\
& \geq \operatorname{Cat}_{\Omega_{\varepsilon^{\alpha}}}\left(\Omega_{\varepsilon^{\alpha}}, T_{c^{\prime} \varepsilon \ln \varepsilon^{-1}} \backslash T_{\varepsilon R}\right),
\end{aligned}
$$

On the other hand, it follows from the definition that

$$
\operatorname{Cat}_{\Omega_{\varepsilon} \alpha}\left(\Omega_{\varepsilon^{\alpha}}, T_{c^{\prime} \varepsilon \ln \varepsilon^{-1}} \backslash T_{\varepsilon R}\right) \geq \operatorname{Cat}_{M_{\varepsilon^{\alpha}}^{k}}\left(M_{\varepsilon^{\alpha}}^{k}, T_{c^{\prime} \varepsilon \ln \varepsilon^{-1}}\right) .
$$

So we have

$$
\#\left\{x: D K(x)=0, x \in K^{c_{\varepsilon, 2}} \backslash K^{c_{\varepsilon, 1}}\right\} \geq \operatorname{Cat}_{M_{\varepsilon^{\alpha}}^{k}}\left(M_{\varepsilon^{\alpha}}^{k}, T_{c^{\prime} \varepsilon \ln \varepsilon^{-1}}\right) .
$$

By Proposition B.4, we know that $M_{\varepsilon^{\alpha}}^{k}$ can not be deformed into $T_{c^{\prime} \varepsilon \ln \varepsilon^{-1}}$. Hence the right hand side of (3.10) is greater than or equal to 1, and thus we have proved the first part of Theorem 1.2.

By Proposition B.5, we know that if $k=2$,

$$
\begin{aligned}
& \#\left\{x: D K(x)=0, x \in K^{c_{\varepsilon, 2}} \backslash K^{c_{\varepsilon, 1}}\right\} \geq \operatorname{Cat}_{M_{\varepsilon^{\alpha}}^{2}}\left(M_{\varepsilon^{\alpha}}^{2}, T_{c^{\prime} \varepsilon \ln \varepsilon^{-1}}\right) \\
\geq & 2 \operatorname{cuplength}\left(M_{\varepsilon^{\alpha}}\right)=2 \operatorname{cuplength}(M) .
\end{aligned}
$$

Thus, the number of two-peak solutions for (1.1) is at least

$$
\frac{1}{2} \#\left\{x: D K(x)=0, x \in K^{c_{\varepsilon, 2}} \backslash K^{c_{\varepsilon, 1}}\right\} \geq \operatorname{cuplength}(M) .
$$

So we complete the proof of Theorem 1.2.

\section{Multipeak solutions COnCEntrating ON The MaXimum SET}

In this section, we assume that $M$ is a local compact maximum set of $V(y)$. Let

$$
\begin{gathered}
\Omega_{\delta}=\left\{x=\left(x_{1}, \ldots, x_{k}\right): x_{j} \in M_{\delta}, j=1, \ldots, k, \frac{\left|x_{i}-x_{j}\right|}{\varepsilon} \geq R, \forall i \neq j\right\}, \\
A_{k, \delta}=\Omega_{\delta} / \sigma_{k} .
\end{gathered}
$$

Define

$$
K_{1}([x])=J\left(\alpha_{\varepsilon}(x), x, v_{\varepsilon}(x)\right), \quad \forall[x] \in A_{k, \delta} .
$$

By (2.6) of Proposition 2.1, $K_{1}([x])$ is well defined in $A_{k, \delta}$. It is not difficult to prove that $A_{k, \delta}$ is a covering space of $\Omega_{\delta}$. As a result, $[x] \in A_{k, \delta}$ is a critical point of $K_{1}$ if and only if $x \in \Omega_{\delta}$ is a critical point of $K$. 
Proof. [Proof of Theorem 1.3]

First, fix $\delta>0$ small such that

$$
\gamma_{1}=: V_{M}-\max _{\partial M_{\delta}} V(x)>0 .
$$

Then take a small positive constant $\gamma$ satisfying $\gamma<\min \left(\gamma_{1}, w(R)\right)$. Let $\delta_{1}>0$ small enough such that

$$
V(x)>V_{M}-\tau \gamma, \quad \forall x \in M_{\delta_{1}},
$$

where $\tau>0$ is a small constant.

Define

$$
c_{\varepsilon}=\varepsilon^{N}\left(k V_{M}^{\frac{p}{p-2}-\frac{N}{2}}-\gamma\right) .
$$

Then it follows from Lemma A.2 that

$$
J\left(\alpha_{\varepsilon}(x), x, v_{\varepsilon}(x)\right)<c_{\varepsilon, k},
$$

if $d\left(x_{i}, M\right)=\delta$ for some $i$, or $\left|x_{i}-x_{j}\right|=\varepsilon R$ for some $i \neq j$. That is

$$
K_{1}([x])<c_{\varepsilon, k}, \quad \forall[x] \in \partial\left(A_{k, \delta}\right) .
$$

Applying Proposition 2.2 to $-K_{1}$, we obtain

$$
\begin{aligned}
& \#\left\{[x]:[x] \in A_{k, \delta}, D K_{1}([x])=0, K_{1}([x]) \geq c_{\varepsilon, k}\right\} \\
& \geq \operatorname{Cat}_{A_{k, \delta}}\left(\left\{K_{1}([x]) \geq c_{\varepsilon, k}\right\}\right) .
\end{aligned}
$$

On the other hand, it is easy to check from (3.1) that

$$
\begin{aligned}
A_{k, \delta_{1}}^{\prime} & =:(\underbrace{M_{\delta_{1}} \times \cdots \times M_{\delta_{1}}}_{k} \backslash \bigcup_{i \neq j}\left\{\frac{\left|x_{i}-x_{j}\right|}{\varepsilon} \geq \frac{1}{\varepsilon^{1 / 2}}\right\}) / \sigma_{k} \\
& \subset\left\{K_{1}([x]) \geq c_{\varepsilon, k}\right\} .
\end{aligned}
$$

Combining (4.1) and (4.2), we obtain

$$
\#\left\{[x]:[x] \in A_{k, \delta}, D K_{1}([x])=0, K_{1}([x]) \geq c_{\varepsilon, k}\right\} \geq \operatorname{Cat}_{A_{k, \delta}}\left(A_{k, \delta_{1}}^{\prime}\right) .
$$

But $A_{k, \delta}$ and $A_{k, \delta_{1}}^{\prime}$ are homotopically equivalent, so we see

$$
\begin{aligned}
& \#\left\{[x]:[x] \in A_{k, \delta}, D K_{1}([x])=0, K_{1}([x]) \geq c_{\varepsilon, k}\right\} \\
\geq & \operatorname{Cat}_{A_{k, \delta}}\left(A_{k, \delta_{1}}^{\prime}\right)=\operatorname{Cat}_{A_{k, \delta}}\left(A_{k, \delta}\right) .
\end{aligned}
$$

Thus we have completed the proof of Theorem 1.3.

Proof. [Proof of Theorem 1.5]

Suppose that $M$ is a connected compact local minimum set. Let $\left(\alpha_{\varepsilon}(x), v_{\varepsilon}(x)\right)$ be the map obtained in Proposition $2.1(k=1)$. Define

$$
\begin{gathered}
K_{3}(x)=J\left(\alpha_{\varepsilon}(x), x, v_{\varepsilon}(x)\right), \forall x \in M_{\gamma}, \\
c_{\varepsilon}^{\prime}=\varepsilon^{N} V_{M}^{\frac{p}{p-2}-\frac{N}{2}} A+\varepsilon^{N} \eta,
\end{gathered}
$$

where $\eta>0$ is a small constant satisfying $\eta<\min _{\partial M_{\gamma}} V(x)-V_{M}$. Then it is easy to check that

$$
K_{3}(x)>c_{\varepsilon}^{\prime}, \quad \forall x \in \partial M_{\gamma},
$$




$$
M \subset K_{3}^{c_{\varepsilon}^{\prime}}
$$

As a result, we have

$$
\#\left\{x: x \in M_{\gamma}, D K_{3}(x)=0, x \in K_{3}^{c_{\varepsilon}^{\prime}}\right\} \geq \operatorname{Cat}_{M_{\gamma}}(M) .
$$

The case that $M$ is a maximum set can be treated in a similar way. So we complete the proof of Theorem 1.5.

\section{Multipeak solution CONCEntrating on Different Sets}

Suppose that $M_{1}$ and $M_{2}$ are two disjoint minimum or maximum sets of $V(y)$. The aim of this section is to construct solution $u$ for (1.1) such that $u$ has $k_{i}$ peaks near $M_{i}, i=1,2$.

Proof. [Proof of Theorem 1.4]

For the simplicity of the notation, we only prove Theorem 1.4 for $l=2$.

For any $\gamma>0$, define

$$
\begin{gathered}
\Omega_{1, \gamma}=\left\{\left(x_{1}, \ldots, x_{k_{1}}\right): x_{i} \in M_{1, \gamma}, \frac{\left|x_{i}-x_{j}\right|}{\varepsilon} \geq R, \forall i \neq j\right\}, \\
\Omega_{2, \gamma}=\left\{\left(x_{k_{1}+1}, \ldots, x_{k_{1}+k_{2}}\right): x_{i} \in M_{2, \gamma}, \frac{\left|x_{i}-x_{j}\right|}{\varepsilon} \geq R, \forall i \neq j\right\}, \\
\Omega_{\gamma}^{*}=\Omega_{1, \gamma} \times \Omega_{2, \gamma} .
\end{gathered}
$$

For any $x \in \Omega_{\gamma}^{*}$, let $\left(\alpha_{\varepsilon}(x), v_{\varepsilon}(x)\right)$ be the map obtained in Proposition 2.1. Define

$$
K(x)=J\left(\alpha_{\varepsilon}(x), x, v_{\varepsilon}(x)\right) .
$$

Since the interaction between the peaks near $M_{1}$ and the peaks near $M_{2}$ is exponentially small, we have

$$
\begin{aligned}
K(x)= & \varepsilon^{N} \sum_{i=1}^{2} k_{i} V_{i}^{\frac{p}{p-2}-\frac{N}{2}} A+\varepsilon^{N} B \sum_{j=1}^{k_{1}}\left(V\left(x_{j}\right)-V_{M_{1}}\right) V_{M_{1}}^{\frac{2}{p-2}-\frac{N}{2}} \\
& +\varepsilon^{N} B \sum_{j=k_{1}+1}^{k_{1}+k_{2}}\left(V\left(x_{j}\right)-V_{M_{2}}\right) V_{M_{2}}^{\frac{2}{p-2}-\frac{N}{2}} \\
& -\sum_{i=1}^{k_{1}-1} \int_{R^{N}}\left(\sum_{j=i+1}^{k_{1}} U_{\varepsilon, x_{j}, M_{1}}\right)^{p-1} U_{\varepsilon, x_{i}, M_{1}} \\
& -\sum_{i=k_{1}+1}^{k_{1}+k_{2}-1} \int_{R^{N}}\left(\sum_{j=i+1}^{k_{1}+k_{2}} U_{\varepsilon, x_{j}, M_{2}}\right)^{p-1} U_{\varepsilon, x_{i}, M_{2}}
\end{aligned}
$$




$$
\begin{aligned}
& +O\left(\sum_{j=1}^{k_{1}+k_{2}} \sum_{m=1}^{[h]} \varepsilon^{N+m}\left|D^{m} V\left(x_{j}\right)\right|\right) \\
& +O\left(\varepsilon^{N} \sum_{1 \leq i<j \leq k_{1}+k_{2}} w^{1+\tau}\left(\frac{\left|x_{i}-x_{j}\right|}{\varepsilon}\right)\right) \\
& +O\left(\varepsilon^{N} \sum_{j=1}^{k_{1}}\left|V\left(x_{j}\right)-V_{M_{1}}\right|^{2}+\varepsilon^{N} \sum_{j=k_{1}+1}^{k_{1}+k_{2}}\left|V\left(x_{j}\right)-V_{M_{2}}\right|^{2}\right) .
\end{aligned}
$$

Case 1. Suppose that both $M_{1}$ and $M_{2}$ are maximun sets. In this case, similar to the proof of Theorem 1.3, using (5.1), we can check

$$
\max _{x \in \partial \Omega_{\delta}^{*}} K(x)<\max _{x \in \Omega_{\delta}^{*}} K(x) .
$$

As a result, the maximum point $x_{\varepsilon}$ of $K(x)$ in $\Omega_{\delta}^{*}$ is an interior point of $\Omega_{\delta}^{*}$, and thus a critical point of $K(x)$.

Case 2. Suppose that both $M_{1}$ and $M_{2}$ are minimum sets with nontrivial topology. Let

$$
\begin{gathered}
c_{\varepsilon, 1}^{\prime}=\varepsilon^{N} \sum_{i=1}^{2} V_{M_{i}}^{\frac{p}{p-2}-\frac{N}{2}} k_{i} A-T \varepsilon^{N+\alpha h}, \\
c_{\varepsilon, 2}^{\prime}=\varepsilon^{N}\left(\sum_{i=1}^{2} V_{M_{i}}^{\frac{p}{p-2}-\frac{N}{2}} k_{i} A+\eta\right), \\
K^{c}=\left\{x: x \in \Omega_{\varepsilon^{\alpha}}^{*}, K(x) \leq c\right\},
\end{gathered}
$$

where $\alpha \in(0,1)$ is a fixed constant close to 1 .

Using (5.1), we see that $K(x)<c_{\varepsilon, 1}^{\prime}$ if $\left|x_{i}-x_{j}\right|=\varepsilon R$ for some $x_{i}, x_{j} \in$ $M_{1, \varepsilon^{\alpha}}$ or $x_{i}, x_{j} \in M_{2, \varepsilon^{\alpha}}$.

Suppose that $x_{1} \in \partial M_{1, \varepsilon^{\alpha}}$. Define $\eta_{1}=\min _{1 \leq i<j \leq k_{1}+k_{2}}\left|x_{i}-x_{j}\right|$. Then, similar to Lemma 3.1, we have $K(x)<c_{\varepsilon, 1}^{\prime}$ if $w\left(\frac{\eta_{1}}{\varepsilon}\right)>L \varepsilon^{\alpha h}$, and $\frac{\partial K(x)}{\partial n}>0$ if $w\left(\frac{\eta_{1}}{\varepsilon}\right) \leq L \varepsilon^{\alpha h}$. So, in order to prove that $K(x)$ has critical point in $K^{c_{2, \varepsilon}} \backslash K^{c_{1, \varepsilon}}$, we just need to prove that $K^{c_{\varepsilon, 2}}$ can not be deformed into $K^{c_{\varepsilon, 1}}$.

It is easy to check $K^{c_{\varepsilon, 2}}=\Omega_{\varepsilon^{\alpha}}^{*}$.

Since

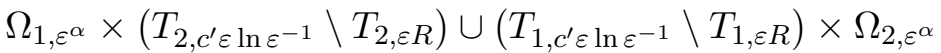

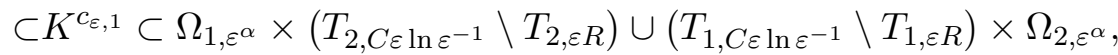

where $T_{l, c}=\cup_{i \neq j}\left\{\left|x_{i}-x_{j}\right| \leq c, x_{i}, x_{j} \in M_{l, \varepsilon^{\alpha}}\right\}, l=1,2, C>0$ is a large constant and $c^{\prime}>0$ is a small constant, we see that if $K^{c_{\varepsilon, 2}}$ could be deformed into $K^{c_{\varepsilon, 1}}$, then $M_{1, \varepsilon^{\alpha}}^{k_{1}} \times M_{2, \varepsilon^{\alpha}}^{k_{2}}$ could be deformed into $T_{1, c^{\prime} \varepsilon \ln \varepsilon^{-1}} \times$ $M_{\varepsilon^{\alpha}, 2}^{k_{2}} \cup M_{\varepsilon^{\alpha}, 1}^{k_{1}} \times T_{2, c^{\prime} \varepsilon \ln \varepsilon^{-1}}$. This is a contradiction to Propostion B.2, since $H_{*}\left(M_{i, \varepsilon^{\alpha}}, T_{i, c^{\prime} \varepsilon \ln \varepsilon^{-1}}\right) \neq 0, i=1,2$. So we have completed the proof of (i) of Theorem 1.4. Using Proposition B.4, we can prove part (ii) of Theorem 1.4 in a similar way. 
Case 3. Suppose that $M_{1}$ is a minimum set and $M_{2}$ is a maximum set. We only consider the case that $M_{1}$ has nontrivial topology. Let

$$
\begin{gathered}
c_{\varepsilon, 1}^{\prime}=\varepsilon^{N} \sum_{i=1}^{2} V_{M_{i}}^{\frac{p}{p-2}-\frac{N}{2}} k_{i} A-T \varepsilon^{N+\alpha h}, \\
c_{\varepsilon, 2}^{\prime}=\varepsilon^{N}\left(\sum_{i=1}^{2} V_{M_{i}}^{\frac{p}{p-2}-\frac{N}{2}} k_{i} A+\eta\right) .
\end{gathered}
$$

Denote

$$
\begin{gathered}
\Omega_{\varepsilon^{\alpha}}^{\prime}=\Omega_{1, \varepsilon^{\alpha}} \times \Omega_{2, \delta}, \\
K^{c}=\left\{x: x \in \Omega_{\varepsilon^{\alpha}}^{\prime}, K(x) \leq c\right\},
\end{gathered}
$$

where $\alpha \in(0,1)$ is a fixed constant close to 1 .

From (5.1), we have $K(x)<c_{\varepsilon, 1}^{\prime}$ for $x \in \Omega_{1, \varepsilon^{\alpha}} \times \partial \Omega_{2, \delta} \cup \partial T_{1, \varepsilon R} \times \Omega_{2, \delta}$.

We claim that if $K(x) \geq c_{1, \varepsilon}^{\prime}$, then

$$
\begin{gathered}
d\left(x_{j}, M_{2}\right) \leq C \varepsilon^{\alpha}, \\
\sum_{1 \leq i<j \leq k_{1}} w\left(\frac{\left|x_{i}-x_{j}\right|}{\varepsilon}\right)+\sum_{k_{1}+1 \leq i<j \leq k_{1}+k_{2}} w\left(\frac{\left|x_{i}-x_{j}\right|}{\varepsilon}\right) \leq C \varepsilon^{\alpha h} .
\end{gathered}
$$

In fact, suppose that $K(x) \geq c_{1, \varepsilon}^{\prime}$. Then we get from (5.1)

$$
\begin{aligned}
& \sum_{1 \leq i<j \leq k_{1}} w\left(\frac{\left|x_{i}-x_{j}\right|}{\varepsilon}\right)+\sum_{k_{1}+1 \leq i<j \leq k_{1}+k_{2}} w\left(\frac{\left|x_{i}-x_{j}\right|}{\varepsilon}\right) \\
+ & \sum_{j=k_{1}+1}^{k_{1}+k_{2}}\left(V_{M_{2}}-V\left(x_{j}\right)\right) \\
+ & O\left(\sum_{j=k_{1}+1}^{k_{1}+k_{2}} \sum_{m=1}^{[h]} \varepsilon^{m} d\left(x_{j}, M_{2}\right)^{h-m}\right) \leq C \varepsilon^{\alpha h} .
\end{aligned}
$$

But by assumption, $V_{M_{2}}-V\left(x_{j}\right) \geq C d\left(x_{j}, M_{2}\right)^{h}$. Thus, (5.4) implies (5.3). Using (5.4) again, we deduce (5.3).

Assume $x_{1} \in \partial M_{1, \varepsilon^{\alpha}}$ and $K(x) \geq c_{1, \varepsilon}^{\prime}$. Similar to Lemma 3.1, using (5.2) and (5.3), we can prove $\frac{\partial K(x)}{\partial n}>0$. So we conclude that if $K(x)$ has no critical point in $K^{c_{\varepsilon, 2}} \backslash K^{c_{\varepsilon, 1}}$, then $K^{c_{\varepsilon, 2}}$ can be deformed into $K^{c_{\varepsilon, 1}}$.

It is easy to check

$$
\begin{gathered}
K^{c_{\varepsilon, 2}}=\Omega_{\varepsilon^{\alpha}}^{\prime}, \\
\Omega_{1, \varepsilon^{\alpha}} \times \partial \Omega_{2, \delta} \cup\left(T_{1, c^{\prime} \varepsilon \ln \varepsilon^{-1}} \backslash T_{1, \varepsilon R}\right) \times \Omega_{2, \delta} \subset K^{c_{\varepsilon, 1}} .
\end{gathered}
$$


On the other hand, if $K(x) \leq c_{1, \varepsilon}^{\prime}$, then

$$
\begin{aligned}
& \sum_{1 \leq i<j \leq k_{1}} w\left(\frac{\left|x_{i}-x_{j}\right|}{\varepsilon}\right)+\sum_{k_{1}+1 \leq i<j \leq k_{1}+k_{2}} w\left(\frac{\left|x_{i}-x_{j}\right|}{\varepsilon}\right) \\
+ & \sum_{j=k_{1}+1}^{k_{1}+k_{2}}\left(V_{M_{2}}-V\left(x_{j}\right)\right) \\
+ & O\left(\sum_{j=k_{1}+1}^{k_{1}+k_{2}} \varepsilon^{m} d\left(x_{j}, M_{2}\right)^{h-m}\right) \geq c \varepsilon^{\alpha h},
\end{aligned}
$$

which implies

$$
\begin{aligned}
& \sum_{1 \leq i<j \leq k_{1}} w\left(\frac{\left|x_{i}-x_{j}\right|}{\varepsilon}\right)+\sum_{k_{1}+1 \leq i<j \leq k_{1}+k_{2}} w\left(\frac{\left|x_{i}-x_{j}\right|}{\varepsilon}\right) \\
& +C \sum_{j=k_{1}+1}^{k_{1}+k_{2}} d\left(x_{j}, M_{2}\right)^{h} \geq c \varepsilon^{\alpha h},
\end{aligned}
$$

Thus, we obtain

$$
K^{c_{\varepsilon, 1}} \subset A=:\left(T_{1, C \varepsilon \ln \varepsilon^{-1}} \backslash T_{1, \varepsilon R}\right) \times \Omega_{2, \delta} \cup \Omega_{1, \varepsilon^{\alpha}} \times B,
$$

where

$$
\begin{aligned}
& B=\left\{x^{\prime}=\left(x_{k_{1}+1}, \ldots, x_{k_{1}+k_{2}}\right) \in \Omega_{2, \delta}: d\left(x_{j}, M_{2}\right) \geq c^{\prime} \varepsilon^{\alpha}, \text { for some } j\right\}
\end{aligned}
$$

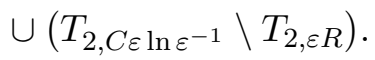

But $A$ can be deformed into $\Omega_{1, \varepsilon^{\alpha}} \times \partial \Omega_{2, \delta} \cup \partial T_{1, \varepsilon R} \times \Omega_{2, \delta}$, so we see that $\Omega_{\varepsilon^{\alpha}}^{\prime}$ can be deformed into $\Omega_{1, \varepsilon^{\alpha}} \times \partial \Omega_{2, \delta} \cup \partial T_{1, \varepsilon R} \times \Omega_{2, \delta}$. But $H_{*}\left(\Omega_{1, \varepsilon^{\alpha}}, \partial T_{1, \varepsilon R}\right) \neq 0$, $H_{*}\left(\Omega_{2, \delta}, \partial \Omega_{2, \delta}\right) \neq 0$. Thus we get a contradiction.

Remark 5.1. If both $M_{1}$ and $M_{2}$ are local maximum sets of $V(y)$, using the same technique as that in section 4 , we see that the number of the solutions with $k_{i}$ peaks near $M_{i}, k_{i} \geq 2, i=1,2$, is at least

$$
\operatorname{Cat}_{A_{1, k_{1}} \times A_{2, k_{2}}}\left(A_{1, k_{1}} \times A_{2, k_{2}}\right),
$$

where

$$
A_{l, k_{1}}=(\underbrace{M_{l, \gamma} \times \cdots \times M_{l, \gamma}}_{k_{i}} \backslash \bigcup_{i \neq j}\left\{\left|x_{i}-x_{j}\right|<d\right\}) / \sigma_{k_{l}}, \quad l=1,2 .
$$

We have $\operatorname{Cat}_{A \times B}(A \times B) \geq \operatorname{cuplength}(A \times B)+1$. On the other hand, it follows the Künneth's formula that cuplength $(A \times B) \geq \operatorname{cuplength}(A)+$ cuplength $(B)$ (We stress here that all the homologies in this paper are with $Z_{2}$-coefficients.). Moreover, by the estimates in Appendix $\mathrm{C}$, we have $\operatorname{cuplength}\left(A_{i, k_{i}}\right) \geq N-1$. Thus we see (1.1) has at least $2 N-1$ different solutions with $k_{i}$ peaks near $M_{i}, i=1,2$. Similarly, if $M_{1}, \cdots, M_{l}$ are disjoint connected compact local maximum sets of $V(x)$, then the number of the solutions with $k_{j}$ peaks near $M_{j}, k_{j} \geq 2$, is least $l(N-1)+1$. 
Remark 5.2. Suppose that $M$ is a connected compact local minimum set of $V(x)$. Using similar technique as that in section 4, we can prove that if $M$ has nontrivial reduced homology, then the number of the $k$-peak solutions with all the peaks near $M$ is at least $\operatorname{Cat}_{A_{k}}\left(A_{k}, \partial T_{d} / \sigma_{k}\right)$, where $T_{d}=\cup_{i \neq j}\left\{\mid x_{i}-\right.$ $\left.x_{j} \mid \leq d, x_{i}, x_{j} \in M_{\gamma}\right\}$ and $d>0$ is a small constant.

\section{Appendix A. Basic estimates}

Lemma A.1. We have

$$
\begin{aligned}
I\left(U_{\varepsilon, z, \bar{V}}\right)= & \varepsilon^{N} \bar{V}^{\frac{p}{p-2}-\frac{N}{2}} A+\varepsilon^{N}(V(z)-\bar{V}) \bar{V}^{\frac{2}{p-2}-\frac{N}{2}} B \\
& +\varepsilon^{N} O\left(\sum_{m=1}^{[h]}\left|D^{m} V(z)\right| \varepsilon^{m}+\varepsilon^{[h]+1}\right),
\end{aligned}
$$

where $A=\left(\frac{1}{2}-\frac{1}{p}\right) \int_{R^{N}} w^{p}$ and $B=\frac{1}{2} \int_{R^{N}} w^{2}$.

Proof. First, noting that $U_{\varepsilon, z, \bar{V}}=\bar{V}^{\frac{1}{p-2}} w\left(\bar{V}^{\frac{1}{2}} \frac{y-z}{\varepsilon}\right)$, we have

$$
\begin{aligned}
I\left(U_{\varepsilon, z, \bar{V}}\right)= & \varepsilon^{N} \bar{V}^{\frac{p}{p-2}-\frac{N}{2}} A+\frac{1}{2} \int_{R^{N}}(V(z)-\bar{V}) U_{\varepsilon, z, \bar{V}}^{2} \\
& +\frac{1}{2} \int_{R^{N}}(V(y)-V(z)) U_{\varepsilon, z, \bar{V}}^{2},
\end{aligned}
$$

But

$$
|V(y)-V(z)|=O\left(\sum_{m=1}^{[h]}|y-z|^{m}\left|D^{m} V(z)\right|+\varepsilon^{[h]+1}\right),
$$

and thus

$$
\int_{R^{N}}(V(y)-V(z)) U_{\varepsilon, z, \bar{V}}^{2}=\varepsilon^{N} O\left(\sum_{m=1}^{[h]}\left|D^{m} V(z)\right| \varepsilon^{m}+\varepsilon^{[h]+1}\right) .
$$

So the result follows from (A.1)-(A.2).

Lemma A.2. We have

$$
\begin{aligned}
& I\left(\sum_{j=1}^{k} U_{\varepsilon, z_{j}, V_{j}}\right) \\
= & A \sum_{j=1}^{k} \varepsilon^{N} V_{j}^{\frac{p}{p-2}-\frac{N}{2}}+B \sum_{j=1}^{k} \varepsilon^{N}\left(V\left(z_{j}\right)-V_{j}\right) V_{j}^{\frac{2}{p-2}-\frac{N}{2}} \\
& -\sum_{i=1}^{k-1} \int_{R^{N}} U_{\varepsilon, z_{i}, V_{i}}\left(\sum_{j=i+1}^{k} U_{\varepsilon, z_{j}, V_{j}}\right)^{p-1} \\
& +\varepsilon^{N} O\left(\sum_{j=1}^{k}\left(\left|V\left(z_{j}\right)-V_{j}\right|^{2}+\sum_{m=1}^{[h]}\left|D^{m} V\left(z_{j}\right)\right| \varepsilon^{m}\right)+\varepsilon^{[h]+1}\right) \\
& +\varepsilon^{N} O\left(\sum_{i \neq j} w^{1+\tau}\left(\frac{\left|z_{i}-z_{j}\right|}{\varepsilon}\right)\right) .
\end{aligned}
$$


Proof. We have

$$
\begin{aligned}
& I\left(\sum_{j=1}^{k} U_{\varepsilon, z_{j}, V_{j}}\right) \\
= & \sum_{j=1}^{k} I\left(U_{\varepsilon, z_{j}, V_{j}}\right)+\frac{1}{2} \sum_{i \neq j} \int_{R^{N}}\left(V(y)-V_{j}\right) U_{\varepsilon, z_{i}, V_{i}} U_{\varepsilon, z_{j}, V_{j}} \\
& +\sum_{i \neq j} \int_{R^{N}} U_{\varepsilon, z_{i}, V_{i}}^{p-1} U_{\varepsilon, z_{j}, V_{j}}+\frac{1}{p} \int_{R^{N}}\left(\left(\sum_{j=1}^{k} U_{\varepsilon, z_{j}, V_{j}}\right)^{p}-\sum_{j=1}^{k} U_{\varepsilon, z_{j}, V_{j}}^{p}\right) .
\end{aligned}
$$

On the other hand, we also have

$$
\begin{aligned}
& \sum_{i \neq j} \int_{R^{N}}\left(V(y)-V\left(z_{j}\right)\right) U_{\varepsilon, z_{i}, V_{i}} U_{\varepsilon, z_{j}, V_{j}} \\
= & O\left(\varepsilon^{N}\left(\left|V\left(z_{j}\right)-V_{j}\right|+\sum_{m=1}^{[h]}\left|D^{m} V\left(z_{j}\right)\right| \varepsilon^{m}+\varepsilon^{[h]+1}\right) \sum_{i \neq j} w\left(\frac{\left|z_{i}-z_{j}\right|}{\varepsilon}\right)\right),
\end{aligned}
$$

and

$$
\begin{aligned}
& \int_{R^{N}}\left(\left(\sum_{j=1}^{k} U_{\varepsilon, z_{j}, V_{j}}\right)^{p}-\sum_{j=1}^{k} U_{\varepsilon, z_{j}, V_{j}}^{p}\right) \\
= & p \sum_{i \neq j} \int_{R^{N}} U_{\varepsilon, z_{i}, V_{i}}^{p-1} U_{\varepsilon, z_{j}, V_{j}}+p \sum_{i=1}^{k-1} \int_{R^{N}} U_{\varepsilon, z_{i}, V_{i}}\left(\sum_{j=i+1}^{k} U_{\varepsilon, z_{j}, V_{j}}\right)^{p-1} \\
& +\varepsilon^{N} O\left(\sum_{i \neq j} w^{1+\tau}\left(\frac{\left|z_{i}-z_{j}\right|}{\varepsilon}\right)\right) .
\end{aligned}
$$

Combining (A.3)-(A.5) and Lemma A.1, we get the desired estimate.

Lemma A.3. For any $v \in E_{\varepsilon, x, k}$, we have

$$
\begin{aligned}
& \left|\sum_{j=1}^{k} \int_{R^{N}}\left(V(y)-V_{j}\right) U_{\varepsilon, x_{j}, V_{j}} v\right| \\
& +\left|\int_{R^{N}}\left(\sum_{j=1}^{k} U_{\varepsilon, x_{j}, V_{j}}^{p-1}-\left(\sum_{j=1}^{k} U_{\varepsilon, x_{j}, V_{j}}\right)^{p-1}\right) v\right| \\
& =O\left(\varepsilon^{\frac{N}{2}}\left(\sum_{j=1}^{k}\left(\left|V\left(x_{j}\right)-V_{j}\right|+\sum_{m=1}^{[h]}\left|D^{m} V\left(x_{j}\right)\right| \varepsilon^{m}\right)+\varepsilon^{[h]+1}\right)\right)\|v\|_{\varepsilon} \\
& +O\left(\varepsilon^{\frac{N}{2}} \sum_{i \neq j} w^{\frac{1+\tau}{2}}\left(\frac{\left|x_{i}-x_{j}\right|}{\varepsilon}\right)\right)\|v\|_{\varepsilon},
\end{aligned}
$$


and

$$
\begin{aligned}
& \left|\sum_{j=1}^{k} \int_{R^{N}}\left(V(y)-V\left(x_{j}\right)\right) U_{\varepsilon, x_{j}, V_{j}} U_{\varepsilon, x_{i}, V_{i}}\right| \\
& +\left|\int_{R^{N}}\left(\sum_{j=1}^{k} U_{\varepsilon, x_{j}, V_{j}}^{p-1}-\left(\sum_{j=1}^{k} U_{\varepsilon, x_{j}, V_{j}}\right)^{p-1}\right) U_{\varepsilon, x_{i}}\right| \\
& =O\left(\varepsilon^{N}\left(\sum_{j=1}^{k}\left(\left|V\left(x_{j}\right)-V_{j}\right|+\sum_{m=1}^{[h]}\left|D^{m} V\left(x_{j}\right)\right| \varepsilon^{m}\right)+\varepsilon^{[h]+1}\right)\right) \\
& +O\left(\varepsilon^{N} \sum_{i \neq j} w^{\frac{1+\tau}{2}}\left(\frac{\left|x_{i}-x_{j}\right|}{\varepsilon}\right)\right) .
\end{aligned}
$$

Proof. The proof of this lemma is similar to that of Lemmas A.1 and A.2, and thus we omit the details.

Lemma A.4. Let $\left(\alpha_{\varepsilon}(x), v_{\varepsilon}(x)\right)$ be the map obtained in Proposition 2.1. Then

$$
\begin{aligned}
\frac{\partial J}{\partial x_{i l}}= & c_{1} \varepsilon^{N} D_{l} V\left(x_{i}\right)-(p-1) \sum_{j \neq i} \int_{\Omega} U_{\varepsilon, x_{i}, V_{i}}^{p-2} U_{\varepsilon, x_{j}, V_{j}} \frac{\partial U_{\varepsilon, x_{i}, V_{i}}}{\partial x_{1 l}} \\
& +O\left(\varepsilon^{N-1} \sum_{i \neq j} e^{\left.-(1+\tau) \frac{\left|x_{i}-x_{j}\right|}{\varepsilon}\right)}\right. \\
& +O\left(\varepsilon^{N-1} \sum_{j=1}^{k} \sum_{m=2}^{[h]}\left|D^{m} V\left(x_{j}\right)\right| \varepsilon^{m}+\varepsilon^{N+h}\right) \\
& +O\left(\varepsilon^{N-1} \sum_{j=1}^{k}\left(\left|V\left(x_{j}\right)-V_{j}\right|^{2}+\sum_{m=1}^{[h]}\left|D^{m} V\left(x_{j}\right)\right|^{2} \varepsilon^{2 m}\right)\right),
\end{aligned}
$$

where $c_{1}>0$ is a constant.

$$
\begin{aligned}
\left\langle\frac{\partial J}{\partial v}, U_{\varepsilon, x_{i}, V_{i}}\right\rangle= & O\left(\varepsilon^{N} \sum_{i \neq j} e^{-(1+\tau) \frac{\left|x_{i}-x_{j}\right|}{\varepsilon}}+\varepsilon^{N+h}\right) \\
& +O\left(\varepsilon^{N} \sum_{j=1}^{k}\left(\left|V\left(x_{j}\right)-V_{j}\right|+\sum_{m=1}^{[h]}\left|D^{m} V\left(x_{j}\right)\right| \varepsilon^{m}\right)\right) .
\end{aligned}
$$

Proof. We have

$$
\begin{aligned}
\frac{\partial J}{\partial x_{i l}}= & \sum_{j=1}^{k} \int_{R^{N}}\left(V(y)-V_{j}\right) U_{\varepsilon, x_{j}, V_{j}} \frac{\partial U_{\varepsilon, x_{i}, V_{i}}}{\partial x_{i l}} \\
& +\sum_{j=1}^{k} \int_{R^{N}} U_{\varepsilon, x_{j}, V_{j}}^{p-1} \frac{\partial U_{\varepsilon, x_{i}, V_{i}}}{\partial x_{i l}}-\int_{R^{N}}\left(\sum_{j=1}^{k} U_{\varepsilon, x_{j}, V_{j}}\right)^{p-1} \frac{\partial U_{\varepsilon, x_{i}, V_{i}}}{\partial x_{i l}}
\end{aligned}
$$




$$
\begin{aligned}
& +O\left(\varepsilon^{N-1}\left(\sum_{i \neq j} e^{-(1+\tau) \frac{\left|x_{i}-x_{j}\right|}{\varepsilon}}+\varepsilon^{2([h]+1)}\right)\right) \\
& +O\left(\varepsilon^{N-1} \sum_{j=1}^{k}\left(\left|V\left(x_{j}\right)-V_{j}\right|^{2}+\sum_{m=1}^{[h]}\left|D^{m} V\left(x_{j}\right)\right|^{2} \varepsilon^{2 m}\right)\right) \\
= & \int_{R^{N}} V(y) U_{\varepsilon, x_{i}, V_{i}} \frac{\partial U_{\varepsilon, x_{i}, V_{i}}}{\partial x_{i l}} \\
& -(p-1) \sum_{j=2}^{k} \int_{\Omega} U_{\varepsilon, x_{i}, V_{i}}^{p-2} U_{\varepsilon, x_{j}, V_{j}} \frac{\partial U_{\varepsilon, x_{i}, V_{i}}}{\partial x_{i l}} \\
& +O\left(\varepsilon^{N-1}\left(\sum_{i \neq j} e^{-(1+\tau) \frac{\left|x_{i}-x_{j}\right|}{\varepsilon}}+\varepsilon^{2([h]+1)}\right)\right) \\
& +O\left(\varepsilon^{N-1} \sum_{j=1}^{k}\left(\left|V\left(x_{j}\right)-V_{j}\right|^{2}+\sum_{m=1}^{[h]}\left|D^{m} V\left(x_{j}\right)\right|^{2} \varepsilon^{2 m}\right)\right) .
\end{aligned}
$$

But

$$
\begin{aligned}
& \int_{R^{N}} V(y) U_{\varepsilon, x_{i}, V_{i}} \frac{\partial U_{\varepsilon, x_{i}, V_{i}}}{\partial x_{i l}}=\frac{1}{2} \int_{R^{N}} \frac{\partial V(y)}{\partial y_{l}} U_{\varepsilon, x_{i}, V_{i}}^{2} \\
= & c_{1} \varepsilon^{N} D_{l} V\left(x_{i}\right)+O\left(\varepsilon^{N-1} \sum_{j=1}^{k} \sum_{m=2}^{[h]}\left|D^{m} V\left(x_{j}\right)\right| \varepsilon^{m}+\varepsilon^{N+[h]+1}\right) .
\end{aligned}
$$

So we see that (A.6) follows from (A.8) and (A.9). (A.7) can be proved similarly.

\section{Appendix B. Some topological Results}

In this section, we give some topological results needed in the proof of our main results. First, we recall the definition for the relative category. See [18].

Definition B.1. Let $Y$ and $A$ be closed subsets of a topological space $X$. Then $\operatorname{Cat}_{X}(A, Y)$ is the least integer $k$ such that $A=\cup_{j=0}^{k} A_{j}$, where, for $0 \leq j \leq k, A_{j}$ is closed and there exists $h_{j} \in C\left([0,1] \times A_{j}, X\right)$ such that

(a) $h_{j}(0, x)=x$ for $x \in A_{j}, 0 \leq j \leq k$,

(b) $h_{0}(1, x) \in Y$ for $x \in A_{0}$ and $h_{0}(t, x)=x$ for $x \in A_{0} \cap Y$ and $t \in[0,1]$,

(c) $h_{j}(1, x)=x_{j}$ for $x \in A_{j}$ and some $x_{j} \in X, 1 \leq j \leq k$.

From the definition, we see $\operatorname{Cat}_{X}(A, Y) \geq 1$ if $A$ can not be deformed into a subset of $Y$ within $X$.

From now on, we assume that all the sets appearing in the propositions of this section are subsets in $R^{m}$ for some positive integer $m$. 
Proposition B.2. Suppose that there are positive integers $p_{1}$ and $q_{1}$ such that $H_{p_{1}}\left(A, A^{\prime}\right) \neq 0, H_{q_{1}}\left(B, B^{\prime}\right) \neq 0$. Then

$$
H_{t}\left(A \times B, A^{\prime} \times B \cup A \times B^{\prime}\right) \neq 0
$$

for some positive integer $t$. In particular, $A \times B$ can not be deformed into $A^{\prime} \times B \cup A \times B^{\prime}$.

Proof. Choose the largest positive integers $p$ and $q$ satisfying $H_{p}\left(A, A^{\prime}\right) \neq 0$ and $H_{q}\left(B, B^{\prime}\right) \neq 0$. Then it follows from Künneth formula [17] that

$$
H_{p+q}\left(A \times B, A^{\prime} \times B \cup A \times B^{\prime}\right)=H_{p}\left(A, A^{\prime}\right) \otimes H_{q}\left(B, B^{\prime}\right) \oplus \text { other group. }
$$

So, $H_{*}\left(A \times B, A^{\prime} \times B \cup A \times B^{\prime}\right) \neq 0$.

Proposition B.3. Let $M$ be a compact n-dimensional manifold with boundary. Then $H_{n}(M, \partial M) \neq 0$.

Proof. This result is well known. See for example [20].

Proposition B.4. Suppose that $M$ has nontrivial reduced homology. Then $H_{*}\left(M^{k}, T\right) \neq 0$, where $T=\cup_{i \neq j}\left\{\left(x_{1}, \cdots, x_{k}\right) \in M^{k}: x_{i}=x_{j}\right\}$.

Proof. For the proof of Proposition B.4, see [15].

Proposition B.5. We have

$$
\text { cuplength }\left(M_{\gamma} \times M_{\gamma}, T_{2}\right) \geq 2 \text { cuplength }\left(M_{\gamma}\right)-1,
$$

where $T_{2}=\left\{x=\left(x_{1}, x_{2}\right) \in M_{\gamma}^{2}:\left|x_{1}-x_{2}\right| \leq d\right\}$ and $d>0$ is a small constant. As a result,

$$
\operatorname{Cat}_{M_{\gamma} \times M_{\gamma}}\left(M_{\gamma} \times M_{\gamma}, T_{2}\right) \geq 2 \text { cuplength }\left(M_{\gamma}\right) .
$$

Proof. For the proof of Proposition B.5, see [15].

\section{Appendix C. Some estimates of the Cuplength}

Let $B\left(R^{N}, k\right)$ be the configuration space of $k$ distinct unordered points of $R^{N}$ defined as follows:

$$
B\left(R^{N}, k\right)=F\left(R^{N}, k\right) / \sigma_{k},
$$

where

$$
F\left(R^{N}, k\right)=\underbrace{R^{N} \times \cdots \times R^{N}}_{k} \backslash \bigcup_{i \neq j}\left\{\left(x_{1}, \cdots, x_{k}\right) \in R^{k N}: x_{i}=x_{j}\right\} .
$$

It is not difficult to check that both $F\left(R^{N}, k\right)$ and $B\left(R^{N}, k\right)$ are path connected if $N \geq 2$. The geometry of such configuration spaces has been extensively studied by topologists in recent years. For sophisticated techniques in this respect, the readers can refer to [9]. In this section, we shall give a lower bound of the category of $B\left(R^{N}, k\right)$, obtained by elementary considerations of the cuplength, relying on the fact that the symmetric group $\sigma_{k}$ contains the alternating group $\hat{\sigma}_{k}$ as a normal subgroup of index 2 . The main result of this section is the following: 
Proposition C.1. If $k=2$ or 3, cuplength $\left(B\left(R^{N}, k\right)\right) \geq N-1$. If $k \geq$ 4 , cuplength $\left(B\left(R^{N}, k\right)\right) \geq 2^{t}-1$, where $t$ is the smallest positive integer satisfying $2^{t}>N-1$.

The proof of Proposition C.1 for $k=2$ is quite easy. In fact, since $B\left(R^{N}, 2\right)$ has the same homotopy type as the real projective space $R P^{N-1}$, we see cuplength $\left(B\left(R^{N}, 2\right)\right)=\operatorname{cuplength}\left(R P^{N-1}\right)=N-1$. To prove Proposition C.1 for $k \geq 3$, we need to do more work.

First, let us recall a general fact in algebraic topology. A two to one covering map $p: \tilde{B} \rightarrow B$ between connected spaces $\tilde{B}$ and $B$ gives rise naturally to a 1-dimensional cohomology class $\theta_{p} \in H^{1}(B)$. One elegant way to describe $\theta_{p}$ is that for any connected $B, H^{1}(B)$ is identifiable with the group of homomorphisms from the fundamental group $\pi_{1}(B)$ to $Z_{2}$. Another way of description is that $\theta_{p}$ is represented by the cocycle whose value on a loop $\omega$ in $B$ is zero or one according to whether or not $\omega$ is the $p$-image of a loop $\tilde{\omega}$ in $\tilde{B}$.

For later purpose, we consider here two examples.

Let $p_{1}: S^{N-1} \rightarrow R P^{N-1}$ be the double covering from a sphere to a real projective space of dimension $N-1$. In this case, $\theta_{p_{1}}$ is the generator of the group $H^{1}\left(R P^{N-1}\right)=Z_{2}$. We know that the cuplength of $\theta_{p_{1}}$ is $N-1$, i.e.,

$$
\underbrace{\theta_{p_{1}} \cup \cdots \cup \theta_{p_{1}}}_{l}=0
$$

if and only if $l \geq N$. See for example [17].

For a further example consider $\tilde{B}=S^{N-1} \times_{T} S^{N-1}$, obtained from $S^{N-1} \times$ $S^{N-1}$ by identifyinig each pair $\left(x_{1}, x_{2}\right)$ with its opposite $\left(-x_{1},-x_{2}\right)$. There is then a double covering map $q: \tilde{B} \rightarrow B$, where $B=R P^{N-1} \times R P^{N-1}, q$ is defined via $q\left(x_{1}, x_{2}\right)=\left(p_{1}\left(x_{1}\right), p_{2}\left(x_{2}\right)\right)$ and $p_{1}, p_{2}$ are the double coverings for the two individual factors respectively. Now

$$
H^{1}(B)=H^{1}\left(R P^{N-1}\right) \oplus H^{1}\left(R P^{N-1}\right)=Z_{2} \oplus Z_{2},
$$

with generators denoted by $\theta_{p_{1}} \otimes 1$ and $1 \otimes \theta_{p_{2}}$ respectively. Because of symmetry, one clearly has

$$
\theta_{q}=\theta_{p_{1}} \otimes 1+1 \otimes \theta_{p_{2}}
$$

in this circumstance.

Consider the iterated cup product:

$$
\theta_{q}^{l}=\underbrace{\theta_{q} \cup \cdots \cup \theta_{q}}_{l} .
$$

We have

Lemma C.2. The iterated cup product $\theta_{q}^{l}$ is zero if and only if $l \geq 2^{t}$, where $2^{t}$ is the smallest power of 2 strictly exceeding $N-1$.

Proof. Remember that we are working mod-2 since $Z_{2}$ is used as coefficient group for cohomology. 
If $l=2^{t}$, then

$$
\begin{aligned}
\theta_{q}^{l} & =\left(\theta_{p_{1}} \otimes 1+1 \otimes \theta_{p_{2}}\right)^{2^{t}} \\
& =\theta_{p_{1}}^{2^{t}} \otimes 1+1 \otimes \theta_{p_{2}}^{2^{t}}=0
\end{aligned}
$$

since $2^{t} \geq N$.

If $l<2^{t}$, write

$$
l=2^{\beta_{1}}+2^{\beta_{2}}+\cdots+2^{\beta_{s}}
$$

with $t>\beta_{1}>\beta_{2}>\cdots>\beta_{s} \geq 0$. Let $l_{1}=2^{\beta_{1}}$ and $l_{2}=l-l_{1}$. Then $l=l_{1}+l_{2}$ with $l_{1}<N, l_{2}<N$ and $\left(\begin{array}{l}l \\ l_{1}\end{array}\right)$ is odd. Note that $\left(\theta_{p_{1}} \otimes 1+1 \otimes \theta_{p_{2}}\right)^{l}$ contains, upon binormial expansion, the nonzero term

$$
\left(\begin{array}{c}
l \\
l_{1}
\end{array}\right)\left(\theta_{p_{1}} \otimes 1\right)^{l_{1}} \cup\left(1 \otimes \theta_{p_{2}}\right)^{l_{2}}=\left(\begin{array}{c}
l \\
l_{1}
\end{array}\right) \theta_{p_{1}}^{l_{1}} \otimes \theta_{p_{2}}^{l_{2}},
$$

which lies in $H^{l_{1}}\left(R P^{N-1}\right) \otimes H^{l_{2}}\left(R P^{N-1}\right)$, a direct summand of $H^{l}\left(R P^{N-1} \times\right.$ $\left.R P^{N-1}\right)$. Thus $\theta_{q}^{l} \neq 0$.

We are now ready to prove Proposition C.1.

Proof. [Proof of Proposition C.1] Define $\tilde{B}\left(R^{N}, k\right)=F\left(R^{N}, k\right) / \hat{\sigma}_{k}$, where $\hat{\sigma}_{k}$ is the alternating group.

Suppose that $k \geq 4$. First, we consider the case $k=2 h$, where $h$ is even. Construct a continuous map

$$
\hat{f}: S^{N-1} \times S^{N-1} \rightarrow \tilde{B}\left(R^{N}, k\right),
$$

by sending $(a, b)$ in $S^{N-1} \times S^{N-1}$ to the orbit of

$$
(a,-a, 2 a,-2 a, \cdots,(h-1) a,-(h-1) a, h b,-h b)
$$

under the $\hat{\sigma}_{k}$-action on $F\left(R^{N}, k\right)$. It can be easily checked that because $h$ is even, $\hat{f}$ sends $(-a,-b)$ to the same $\hat{\sigma}_{k^{-}}$orbit. Therefore, it induces a map $\tilde{f}: S^{N-1} \times_{T} S^{N-1} \rightarrow \tilde{B}\left(R^{N}, k\right)$. Bringing in the double coverings $p$ and $q$, we form a commutative diagram:

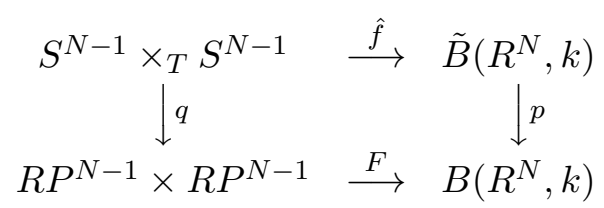

where $F$ is a uniquely induced map. Note once more that for any point $u$ in $R P^{N-1} \times R P^{N-1}$ and its image point $v=F(u)$ in $B\left(R^{N}, k\right)$, the restriction of $\tilde{f}$ maps $q^{-1}(u)$ to $p^{-1}(v)$ bijectively. By the natural way $\theta_{p}$ and $\theta_{q}$ arise from their respective double coverings, we see that for the induced homomorphism

$$
F^{*}: H^{1}\left(B\left(R^{N}, k\right)\right) \rightarrow H^{1}\left(R P^{N-1} \times R P^{N-1}\right),
$$

$F^{*}\left(\theta_{p}\right)$ naturally equals $\theta_{q}$.

By Lemma C.2, $\theta_{q}^{l} \neq 0$ if $l$ is less than $2^{t}$, the smallest power of 2 exceeding $N-1$. Since $F^{*}$ is a homomorphism of cohomology rings, the same must be true for $\theta_{p}^{l}$. Thus we have exhibited in $B\left(R^{N}, k\right)$ a nonzero cupproduct of length $2^{t}-1$, and our claim follows. 
If $k=2 h+j$, where $h$ is even and $1 \leq j \leq 3$, we can use the same proof by modifying the map $\hat{f}$ into the one which sends $(a, b)$ and $(-a,-b)$ to the $\hat{\sigma}_{k}$-orbit of

$$
\left(a,-a, 2 a,-2 a, \cdots,(h-1) a,-(h-1) a, h b,-h b, c_{1}, \cdots, c_{j}\right)
$$

instead, where $c_{1}, \cdots, c_{j}$ are $j$ distinct points fixed in $R^{N}$, each having distance greater than $h+1$ from the origin.

Suppose that $k=3$. Define a map $\hat{f}: S^{N-1} \rightarrow \tilde{B}\left(R^{N}, 3\right)$ by sending $a \in S^{N-1}$ to the orbit of $(a,-a, 0)$ in $F\left(R^{N}, 3\right)$ under the $\hat{\sigma}_{3}$-action. We have the following commutative diagram:

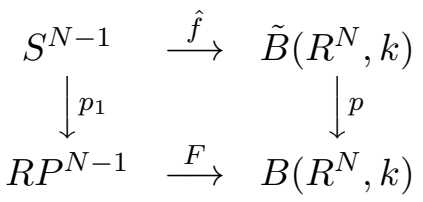

where $F$ is a uniquely induced map. Since the nonzero element $\theta_{p_{1}} \in$ $H^{1}\left(R P^{N-1}\right)$ has cuplength $N-1$ (see the first example above), we can prove in a similar way as above that $\theta_{p}^{N-1} \neq 0$. So we have proved Proposition C.1 for the case $k=3$. As we mentioned before, the Proposition C. 1 is quite easy to prove if $k=2$.

As a direct consequence of Proposition C.1, we have

Proposition C.3. If $k \geq 4$, we have Cat $_{A_{k}}\left(A_{k}\right) \geq 2^{t}$, where $t$ is the smallest positive integer satisfying $2^{t}>N-1$. If $k=2,3$, we have $\operatorname{Cat}_{A_{k}}\left(A_{k}\right) \geq N$. Here

$$
A_{k}=(\underbrace{M_{\gamma} \times \cdots \times M_{\gamma}}_{k} \backslash \bigcup_{i \neq j}\left\{\left|x_{i}-x_{j}\right|<d\right\}) / \sigma_{k} .
$$

Proof. Let $x_{0} \in M_{\gamma}$ and let $\delta>0$ be so small that $B_{\delta}\left(x_{0}\right) \subset M_{\gamma}$. We also let

$$
V_{k}=(\underbrace{B_{\delta}\left(x_{0}\right) \times \cdots \times B_{\delta}\left(x_{0}\right)}_{k} \backslash \bigcup_{i \neq j}\left\{\left|x_{i}-x_{j}\right| \leq d\right\}) / \sigma_{k},
$$

Then $B\left(R^{N}, k\right)$ and $V_{k}$ are homotopically equivalent. So

$$
\begin{aligned}
& \operatorname{Cat}_{A_{k}} A_{k} \geq \operatorname{Cat}_{B\left(R^{N}, k\right)}\left(V_{k}\right) \\
= & \operatorname{Cat}_{B\left(R^{N}, k\right)}\left(B\left(R^{N}, k\right)\right) \geq \operatorname{cuplength}\left(B\left(R^{N}, k\right)\right)+1 .
\end{aligned}
$$

So Proposition C.3 follows from Proposition C.1.

\section{REFERENCES}

[1] A. Bahri, Critical points at infinity in some variational problems, Pitman Research Notes in Mathematics Series, 182, Longman Scientific, 1989.

[2] A. Bahri and J. M. Coron, On a nonlinear elliptic equation involving the Sobolev exponent: the effect of the topology of the domain, Comm. Pure Appl. Math.41 (1988), 253-294.

[3] V. Benci and G. Cerami, The effect of the domain topology on the number of positive solutions of nonlinear problems, Arch. Rational Mech. Anal. 114 (1991), 79-93. 
[4] V. Benci, G. Cerami and D. Passaseo, On the number of positive solutions of some nonlinear elliptic problems, in Nonlinear Analysis, A Tribute in Honour of G. Prodi, (A. Ambrosetti and A. Marino eds.), Quaderni, Sc. Norm. Sup., Pisa, 93-107, 1991.

[5] D. Cao, E. N. Dancer, E. Noussair and S. Yan, On the existence and profile of multipeaked solutions to singularly perturbed semilinear Dirichlet problems, Discrete Contin. Dynam. Systems, 2 (1996), 221-236.

[6] D. Cao and E. Noussair, Multiplicity of positive and nodal solutions for nonlinear elliptic problems on $R^{N}$, Ann. Inst. H. Poincaré Anal. Non Linéaire, 13 (1996), 567588 .

[7] D. Cao, E. Noussair and S. Yan, Solutions with multiple peaks for nonlinear elliptic equations, Proc. Roy. Soc. Edinburgh Sect. A, 129 (1999), 235-264.

[8] D. Cao, E. Noussair and S. Yan, Existence and uniqueness results on single-peaked solutions of a nonlinear problem, Ann. Inst. H. Poincaré Anal. Non Linéaire, 15 (1998), 73-111.

[9] F. Cohen, The homology of $C_{n+1}$-space, $n \geq 0$, Lecture Notes in Mathematics, 533, 207-351.

[10] E. N. Dancer, Superlinear problems on domains with holes of asymptotic shape and exterior problem, Math. Z. 229 (1998), 475-491.

[11] E. N. Dancer, Some slightly subcritical or slightly supercritical problems, preprint.

[12] E. N. Dancer and J. Wei, On the effect of domain topology in a singular perturbation problem, Topol. Methods Nonlinear Anal. 11 (1998), 227-248.

[13] E. N. Dancer and S. Yan, Multipeak solutions for a singularly perturbed Neumann problem, Pacific J. Math. 189 (1999), 241-262.

[14] E. N. Dancer and S. Yan, Singularly perturbed elliptic problem in exterior domains, Differential Integral Equations, to appear.

[15] E. N. Dancer and S. Yan, A singularly perturbed elliptic problem in bounded domains with nontrivial topology, Adv. Differential Equations, 4 (1999), 347-368.

[16] M. del Pino and P. Felmer, Multi-peak bound states for nonlinear Schrödinger equations, Ann. Inst. H. Poincaré Anal. Non Linéaire, to appear.

[17] A.Dold, Lectures on algebraic topology, Springer-Verlag, Berlin, 1972.

[18] G. Fournier and M. Willem, Relative category and the calculus of variations, in Variational Methods, Progress in Nonlinear Differential Equations and Their Applications, (H. Berestycki, J. M. Coron and I. Ekeland eds.), Birkhäuser, 1990, 95-104.

[19] B. Gidas, W. M. Ni and L. Nirenberg, Symmetry and related properties via the maximum principle, Comm. Math. Phys. 68 (1979), 209-243.

[20] M. J. Greenberg and J. R. Harper, Algebraic topology, a first course, Addison-Wesley, 1981.

[21] C. Gui, Existence of multi-bump solutions for nonlinear Schrödinger equations via variational method, Comm. Partial Differential Equations, 21 (1996), 787-820.

[22] Y. Y. Li, On a singularly perturbed elliptic equation, Adv. Differential Equations, 2 (1997), 955-980.

[23] E. S. Noussair and S. Yan, On the positive multipeak solutions of a nonlinear elliptic problem, Proc. London Math. Soc., to appear.

[24] O. Rey, The role of the Green's function in a non-linear elliptic equation involving the critical Sobolev exponent, J. Funct. Anal. 89 (1990), 1-52.

[25] S. Yan, On the number of interior multipeak solutions for singularly perturbed Neumann problems, Topol. Methods Nonlinear Anal. 12 (1998), 61-68.

\section{School of Mathematics and Statistics \\ UNIVERSITY OF SYDNEY \\ NSW 2006, AUSTRALIA}

E-mail address: normd@maths.usyd.edu.au 
Department of Mathematics

UNIVERsity of British COlumbia

VANCOUVER, CANADA

E-mail address: lam@math.ubc.ca

School of Mathematics and Statistics

UNIVERSITY OF SYDNEY

NSW 2006, AUSTRALIA

E-mail address: shusen@maths.usyd.edu.au 


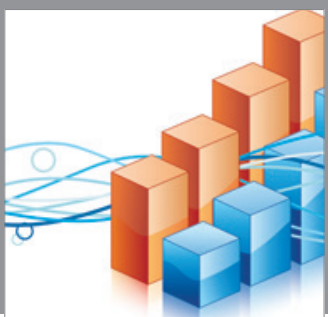

Advances in

Operations Research

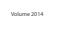

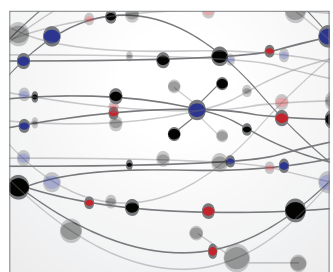

\section{The Scientific} World Journal
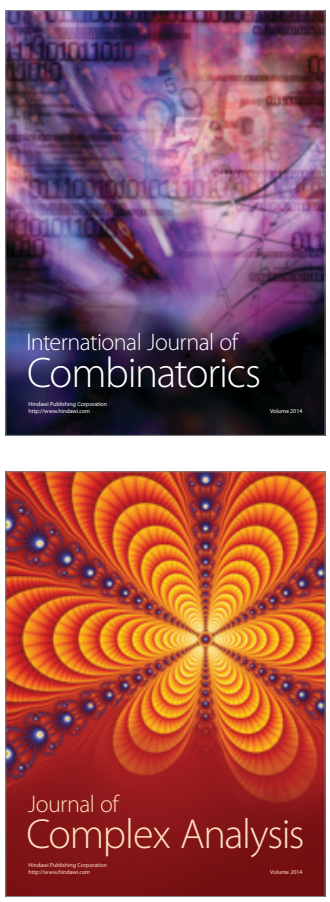

International Journal of

Mathematics and

Mathematical

Sciences
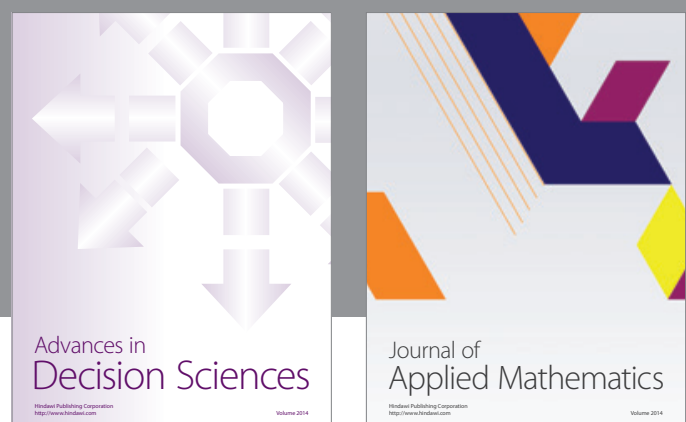

Journal of

Applied Mathematics
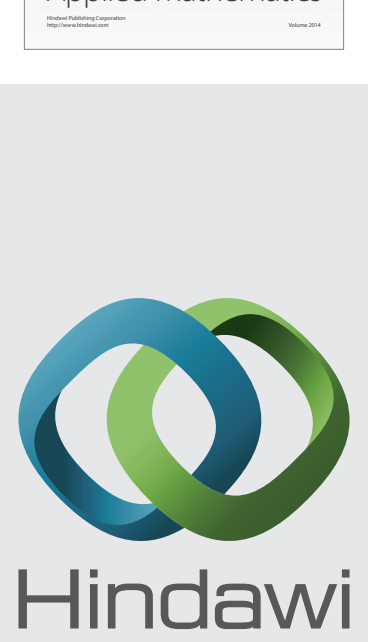

Submit your manuscripts at http://www.hindawi.com
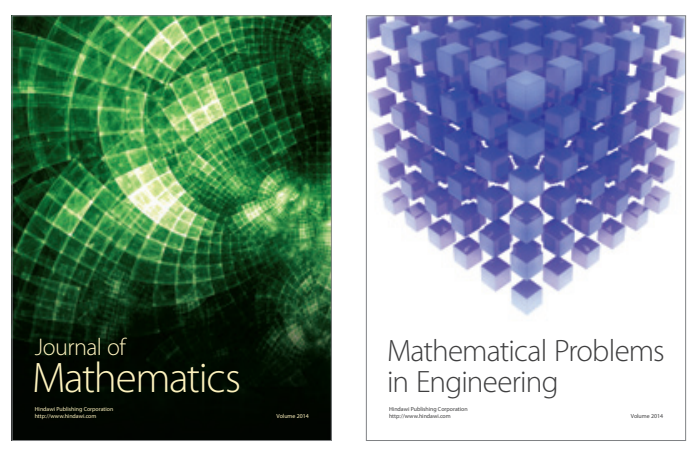

Mathematical Problems in Engineering
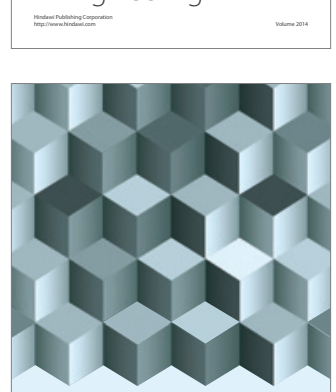

Journal of

Function Spaces
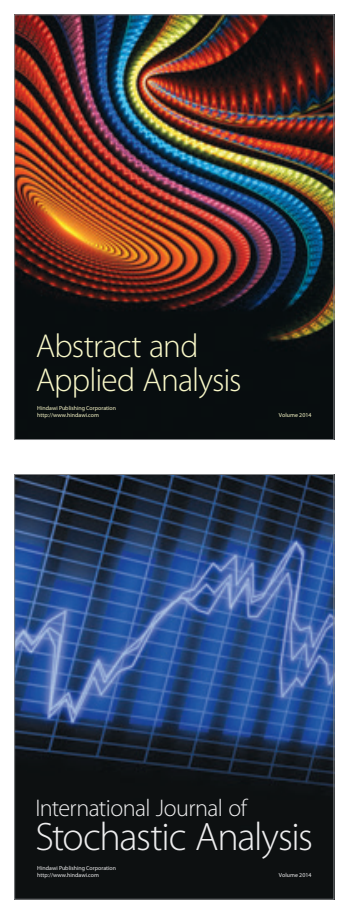

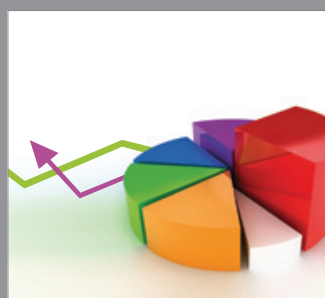

ournal of

Probability and Statistics

Promensencen
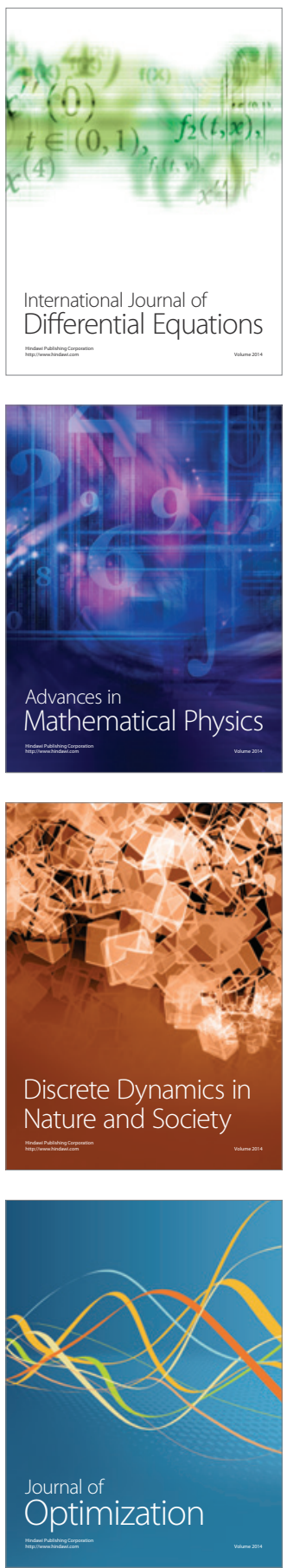